\title{
SST/CRISP observations of convective flows in a sunspot penumbra
}

\author{
G. B. Scharmer ${ }^{1,2}$ and V. M. J. Henriques ${ }^{1.2}$

\begin{abstract}
1 Institute for Solar Physics, Royal Swedish Academy of Sciences, AlbaNova University Center, 10691 Stockholm, Sweden
2 Stockholm Observatory, Dept. of Astronomy, Stockholm University, AlbaNova University Center, 10691 Stockholm, Sweden e-mail: scharmer@astro.su.se
\end{abstract}

Received 6 September 2011 / Accepted 23 January 2012

\section{ABSTRACT}

\begin{abstract}
Context. Recent discoveries of intensity correlated downflows in the interior of a sunspot penumbra provide direct evidence for overturning convection, adding to earlier strong indications of convection from filament dynamics observed far from solar disk center, and supporting recent simulations of sunspots.

Aims. Using spectropolarimetric observations obtained at a spatial resolution approaching 0.'1 with the Swedish 1-m Solar Telescope (SST) and its spectropolarimeter CRISP, we investigate whether the convective downflows recently discovered in the $\mathrm{C}_{\mathrm{I}}$ line at $538.03 \mathrm{~nm}$ can also be detected in the wings of the Fe I line at $630.15 \mathrm{~nm}$.

Methods. We make azimuthal fits of the measured LOS velocities in the core and wings of the $538 \mathrm{~nm}$ and $630 \mathrm{~nm}$ lines to disentangle the vertical and horizontal flows. To investigate how these depend on the continuum intensity, the azimuthal fits are made separately for each intensity bin. By using spatially high-pass filtered measurements of the LOS component of the magnetic field, the flow properties are determined separately for magnetic spines (relatively strong and vertical field) and inter-spines (weaker and more horizontal field). Results. The dark convective downflows discovered recently in the $538.03 \mathrm{~nm}$ line are evident also in the $630.15 \mathrm{~nm}$ line, and have similar strength. This convective signature is the same in spines and inter-spines. However, the strong radial (Evershed) outflows are found only in the inter-spines.

Conclusions. At the spatial resolution of the present SST/CRISP data, the small-scale intensity pattern seen in continuum images is strongly related to a convective up/down flow pattern that exists everywhere in the penumbra. Earlier failures to detect the dark convective downflows in the interior penumbra can be explained by inadequate spatial resolution in the observed data.
\end{abstract}

Key words. sunspots - convection - magnetic fields - magnetohydrodynamics (MHD)

\section{Introduction}

The origin of sunspot penumbral filamentary structure and strong horizontal flow (Evershed 1909), their complex magnetic field topology, and the mechanism that transports energy to the visible surface represent some of the most longstanding puzzles in astrophysics. An early proposal for explaining this filamentary structure was in terms of convection rolls (Danielson 1961b), based on sunspot photographs recorded during the 1959 Stratoscope flights (Danielson 1961a). Initial support for this interpretation came from spectroscopic observations at low spatial resolution of a sunspot at $16^{\circ}$ heliocentric distance (Beckers \& Schröter 1969), demonstrating a correlation of 29-39\% between the continuum intensity and LOS velocity measured in the line wings of the Fe r line at $557.6 \mathrm{~nm}$. Later observations did not give consistent support for strong such correlations (e.g., Wiehr \& Stellmacher 1989; Lites et al. 1990). Johannesson (1993) found a generally weak or non-existent correlation between the continuum intensity and the flow field, with only a tendency for bright structures to correspond to local blue shifts on both sides of the spot, while Sanchez Almeida et al. (1993) claimed clear evidence of such a correlation, based on $21 \mathrm{Fe}_{\mathrm{I}} 630.15 \mathrm{~nm}$ and $630.25 \mathrm{~nm}$ spectra recorded with an 0.5 spectrograph slit. More recent observations with the Swedish 1-m Solar Telescope (SST) at much higher spatial resolution of part of a sunspot at $35^{\circ}$ heliocentric distance (Sánchez Almeida et al. 2007) demonstrated a clear correlation between intensity and vertical velocity in the sense expected for convection. We note, however, that none of the above observations demonstrated evidence that the dark penumbral filaments actually have absolute downward flows, as required for these flows to be identified as convective. We finally note that indirect evidence for penumbral convection was found from analysis of proper motions of small-scale intensity structures, obtained by employing local cross correlation techniques to a time series of SST G-band images (Márquez et al. 2006). A relevant question is to what extent velocities inferred from intensity patterns reflect true flows.

In parallell, attempts were made to explain the Evershed flow, and to characterize its relation to bright and dark filamentary fine structure. A highly influential explanation was in terms of siphon flows in flux tubes (Meyer \& Schmidt 1968). This apparently triggered the development of models and numerical simulations of flows in flux tubes for a period of over 30 years (Thomas \& Montesinos 1993; Solanki \& Montavon 1993; Schlichenmaier et al. 1998; Schlichenmaier 2002). Support for such models came from observations at about 0.7 spatial resolution showing bright upflows in the inner penumbra and dark downflows in the outer penumbra (Schlichenmaier \& Schmidt 1999; Schmidt \& Schlichenmaier 2000). These authors also found an overall correlation between brightness and vertical velocity that can be interpreted as an indication of convection. However, throughout the inner two thirds of the penumbra observed, both the locally bright and dark component of the penumbra on the average showed only upflows. 
An important step toward our understanding of the fine structure of penumbral magnetic fields came with the discovery of broadband circular polarization in sunspots (Illing et al. 1974a,b), implying the simultaneous existence of strong lineof-sight (LOS) gradients in the magnetic field and flow velocity (Sanchez Almeida \& Lites 1992). The inferred strong gradients appeared to imply extremely strong currents and curvature forces in the visible layers of penumbrae (Sanchez Almeida \& Lites 1992; Solanki et al. 1993; Solanki \& Montavon 1993) (note however, that strong gradients in the magnetic field do not necessarily imply strong forces: potential fields above nearly fieldfree gaps give rise to strong LOS gradients above the gaps, but are force-free; Spruit \& Scharmer 2006). This (apparent) problem was addressed by the uncombed penumbra model (Solanki \& Montavon 1993), based on the assumed existence of slender nearly horizontal flux tubes with constant magnetic field within the flux tube (no volume currents). The flux tubes were assumed to be elevated above the penumbral photosphere and carrying the Evershed flow, and to be embedded in a more vertical background magnetic field. Various calculations and inversions (Solanki \& Montavon 1993; Martínez Pillet 2000; Schlichenmaier \& Collados 2002; Bellot Rubio et al. 2003, 2004; Borrero et al. 2007; Tritschler et al. 2007) based on this and similar simplified flux tube models gave consistency with the spectropolarimetric data, at that time rarely reaching better than $1^{\prime \prime}$ spatial resolution. An alternative explanation of observed penumbral Stokes spectra was given by Sánchez Almeida $(2005,2006)$. He postulated the existence of micro-structured magnetic field and velocity structures (represented as two optically thin magnetic components) and used a special inversion code (Sanchez Almeida 1997) to test this conjecture. Fitting his observed spectra required the existence of simultaneous upflows and downflows throughout the penumbra, which can be interpreted as evidence of convection. However, these inversions were constrained by the temperature being equal for the two magnetic components, which excludes any convective energy flux. A surprising result of the inversions is the existence of opposite polarity field everywhere in the penumbra. We note that the inversions constrain the magnetic fields and flows in the two magnetic components to be strictly parallell, such that any mixture of upflows and downflows must be associated with mixed polarity field, and vice versa.

It was soon evident that a flow in a slender flux tube cannot carry the energy needed to compensate the energy losses throughout the entire radial extent of a penumbra (Schlichenmaier \& Solanki 2003). This and other problems of flux tube models were high-lighted by Spruit \& Scharmer (2006). They concluded that the origin of the filamentary structure and the large fluctuations in field strength and magnetic field inclination across penumbral filaments is not embedded flux tubes but must be convection in (nearly) field-free gaps $b e$ low the visible surface ${ }^{1}$, resolving the heat flux problem. Simple potential field and magnetohydrostatic models (Scharmer \& Spruit 2006) of such "gaps" demonstrated that large fluctuations in magnetic field strength and inclination are unavoidable

\footnotetext{
${ }^{1}$ We make two remarks. First, as in the quiet Sun, the penumbral photosphere is convectively stable (Rempel 2011), and the flows seen in the line forming layers represent the overshoot of this convection. Gaps are thus expected primarily in the invisible layers just below the photosphere, and to what extent they are field-free can probably only be investigated by means of simulations. Second, the gaps are not expected to be strictly field-free, but rather to have sufficiently reduced field strengths to allow the overturning convective flows to occur (Nordlund \& Scharmer 2010; Spruit et al. 2010).
}

consequences of such gaps, but without giving rise to any magnetic forces, and that the magnetic field should be much more horizontal above than outside the gaps. The model also explained the penumbral dark cores (Scharmer et al. 2002) as due to a strongly warped $\tau=1$ surface caused by the strong azimuthal fluctuations in gas pressure (induced by the strong fluctuations in magnetic field strength) combined with an overall drop in temperature with height ${ }^{2}$. While the convective gap model did not at that time explain the origin of the Evershed flow, it was evident that this flow can only exist in the convective gaps or immediately above them, where the field is nearly horizontal (Spruit \& Scharmer 2006).

Theoretical support for the convective origin of penumbral fine structure came with the first MHD simulation of penumbral fine structure (Heinemann et al. 2007). While limited to a thin slice of a quite small modelled sunspot, these simulations demonstrated convection with strongly reduced (but far from zero) field strength, a systematic (but weak) radial outflow in gaps, dark cores above short penumbral filaments, an inward migration of the penumbral structure toward the penumbra (as observed in movies), and a moat flow outside the sunspot. Based on these simulations, the Evershed flow was explained as being identical to the horizontal component of penumbral convection (Scharmer et al. 2008b). Simulations of much larger sunspots and with higher grid resolution (Rempel et al. 2009a,b; Rempel 2011) demonstrated a higher degree of realism, with much longer filaments and stronger Evershed flows. Detailed analysis of the simulation data has clarified some of the properties and driving mechanisms of the penumbral convection and Evershed flow (Rempel 2011). Remarkably, the length scales, mass fluxes and rms velocities of penumbral convective flows are quite similar to those of field-free convection in the quiet Sun (Rempel et al. 2009a).

Strong evidence for penumbral convection came from observations of filamentary dynamics in sunspots located well away from disk center. Ichimoto et al. (2007b) found "twisting motions" in filaments from space-time plots along lines crossing filaments in the inner penumbra. Further evidence for convection (interpreted as convective rolls) in penumbral filaments in SST observations of a sunspot at $40^{\circ}$ heliocentric distance was reported by Zakharov et al. (2008). More recently, evidence for penumbral convection was observed in a sunspot located at $58^{\circ}$ heliocentric distance (Spruit et al. 2010). These authors also pointed out that the tilted "striations", seen in the filaments are similar to those observed in faculae close to the limb and reproduced in MHD simulations (Carlsson et al. 2004), and most likely outline magnetic field lines.

Whereas the convective origin of the penumbral structure and Evershed flow thus seems quite clear from a theoretical perspective, and also was receiving strong support from observations, the accumulating evidence for penumbral convection was not readily accepted. The main objection is the absence of direct observational evidence for convective downflows inside the penumbra. Franz \& Schlichenmaier (2009) and Franz (2011) analyzed spectropolarimetric data from Hinode, recorded from sunspots close to disk center and found no indications of convection in penumbral filaments. However, Franz (2011) actually measured a correlation coefficient for small-scale (high-pass filtered) intensity and velocity fluctuations of up to $-53 \%$ in the

\footnotetext{
2 This is very similar to what is seen in faculae and bright points where locations of strong magnetic field correspond to a locally depressed $\tau=$ 1 surface, leading to enhanced intensity due to the higher temperatures in the deeper layers (Scharmer 2009).
} 
inner parts of one penumbra in the Fe $\mathrm{I} 630.15 \mathrm{~nm}$ line (hereafter referred to as "6301 line"), but considered this degree of correlation as being too small to be significant.

While clear evidence for convection thus appears to be missing (or, is claimed to be missing) in spectropolarimetric Hinode data, the Swedish 1-m Solar Telescope (SST) provides clear such evidence. We recently (Scharmer et al. 2011) made observations in the $C_{\text {I }} 538.03 \mathrm{~nm}$ line (hereafter referred to as the "5380 line"), formed very close to the visible photosphere (Schlichenmaier \& Schmidt 1999), where (overshooting) convective flows are expected to be significantly stronger than at the heights of formation of the 6301 line. The spatial resolution of this SST data is more than 2.3 times higher than that of the spectropolarimetric Hinode data, representing a major improvement in our ability to resolve penumbral filamentary structure and flows. By making azimuthal fits to separately determine the variation of radial (horizontal) and vertical velocities with intensity, clear evidence of a correlation between intensity and vertical velocity was found in the sense expected for convection. In addition, the darkest penumbral features in the interior penumbra ${ }^{3}$ show downflows up to about $1 \mathrm{~km} \mathrm{~s}^{-1}$ while the brightest features show upflows of several $\mathrm{km} \mathrm{s}^{-1}$ (Scharmer et al. 2011). Additional support for the existence of convective downflows was recently found from analysis of other SST data in the 5380 line (Joshi et al. 2011). Using simulated SST observations based on numerical simulations of Rempel et al. (2009b), Bharti et al. (2011) recently confirmed that convective downflows should be observable with the 5380 line at the spatial resolution of the SST, while such downflows would be more difficult to observe in the Fe $709.1 \mathrm{~nm}$ line, explaining in part the failure of Bellot Rubio et al. (2010) in detecting such flows in this line. The other difficulty in detecting vertical flows from their data is the contamination of the LOS velocity from the strong horizontal Evershed flows, due to the 5.4 heliocentric distance (Bharti et al. 2011).

In the present paper we extend the analysis of the observations reported by Scharmer et al. (2011) to include Doppler and magnetic field measurements made in the 6301 line, recorded nearly simultaneously with the recently published 5380 data. We demonstrate that the convective signatures found in the 5380 line also are obvious in the wings of the 6301 line, although with reduced velocity amplitudes, and that the dark penumbral features in the interior penumbra on the average are associated with (convective) downflows also when observed in the 6301 line. We also find that the convective signatures are the same in magnetic spines and inter-spines (Lites et al. 1993), but the strong radial outflows are seen only in the inter-spines. In Sect. 2 of this paper, we describe the observations, data processing and methods of data analysis. Correlations between continuum intensities and radial and vertical velocities obtained from azimuthal fits of LOS velocities measured in the 5380 and 6301 lines are compared and discussed in Sect. 3. Finally, in Sect. 4 we discuss the results and draw conclusions with respect to models.

\section{Observations and data processing}

The data were recorded with the SST (Scharmer et al. 2003a) using the CRisp Imaging SpectroPolarimeter (CRISP; Scharmer 2006; Scharmer et al. 2008a). The SST is an un-obscured 1-m aperture telescope that presently employs low-order (37 electrode) adaptive optics (Scharmer et al. 2003b) to achieve nearly

\footnotetext{
3 Here and in the following we use the term "interior penumbra" to mean the part of the penumbra that is well away from both the inner (umbral) and outer (quiet Sun) boundaries.
}

diffraction limited resolution in excellent seeing at wavelengths as short as $390 \mathrm{~nm}$. Of importance in explaining the excellent data quality is the use of multiple short $(17 \mathrm{~ms})$ exposures that are post-processed with an elaborate image reconstruction technique (van Noort et al. 2005) to compensate residual loworder aberrations left by the AO system. CRISP is a dual FabryPerot filter system with the low-resolution etalon having reduced reflectivity in order to mitigate the effects of cavity errors (variations in separation between the reflecting surfaces of the etalons). The telecentric mounting of the etalons gives wavelength shifts of the transmission peak over the FOV, but the low reflectivity of the low-resolution etalon gives a passband that is wide enough to accommodate the relative wavelength shifts between the two etalons, such that the wavelength transmission profile shows only small variations in its shape over the FOV. The spectral resolution of CRISP is modest, about $4.4 \mathrm{pm}$ at $538 \mathrm{~nm}$ and $6 \mathrm{pm}$ at $630 \mathrm{~nm}$, in order to allow fast tuning of spectral lines at high signal-to-noise and without spectral undersampling. To support image reconstruction and co-alignment between images recorded at different wavelengths, the setup includes one CCD camera that records broad-band images through the CRISP pre-filter used to select a particular spectral line. To enable polarimetric measurements with low levels of seeinginduced cross-talk from Stokes $I$ to $Q, U$, and $V$ there are two cameras at the CRISP final focal plane, with the light divided between the two cameras by a polarizing beam splitter. Polarization modulation is made with two tunable liquid crystals (LC's) with their fast axes at $0^{\circ}$ and $45^{\circ}$ with respect to the vertical axis on the optical table. The LC's have sufficient stroke to allow full Stokes measurements over the entire wavelength range of CRISP (from about $510 \mathrm{~nm}$ to $860 \mathrm{~nm}$ ).

The present data were recorded on 23 May 2010 at 14:11 UT from a reasonably regular sunspot at $15^{\circ}$ heliocentric distance. The data consists of three nearly simultaneous (the time difference between the 5380 and 6301 line scans is 13 s) spectral scans of the 5380 line, the 6301 line, and the Fe I at $630.25 \mathrm{~nm}$ (hereafter the "6302 line"). Due to the telluric blend in the red wing of the 6302 line preventing measurements of the bisector wavelength shifts close to the continuum, we will not discuss the 6302 data in the present paper. The images were recorded at an image scale of 0 .' $^{\prime} 059$ per pixel. After image processing, co-alignment of the images recorded at the three different wavelengths and removing the apodized part of the FOV near the edges, the science data consisted of $881 \times 881$ pixels covering $52^{\prime \prime} \times 52^{\prime \prime}$, shown in Figs. 1 and 2. The 5380 data consist of a total of 960 images sampled with CRISP at 23 wavelengths in typical steps of $2.8 \mathrm{pm}$ plus 480 broad-band images. The 6301 (and 6302) data consist of a total of 600 images sampled at 15 wavelengths in steps of $4.4 \mathrm{pm}$ and 320 broadband images.

Figure 1 shows the $538 \mathrm{~nm}$ continuum image (top) and the 5380 center-of gravity (COG) line-of-sight (LOS) velocity of the entire field-of-view (FOV) observed, Fig. 2 shows the LOS magnetic field obtained with the COG method (Rees \& Semel 1979; Cauzzi et al. 1993). The high-lighted region around most of the outer boundary of the FOV defines the area used as quiet Sun reference (although about a few per cent of this area obviously is covered with strong field), the high-lighted area in the sunspot outlines what we refer to as the "interior penumbra", which is the main target of our analysis. Figures 3 and 4 show all measured quantities within sub-fields (marked with dark rectangles in Fig. 1) close to the limb-side and disk center-side parts of the penumbra.

We note that Fig. 2 shows very little evidence of the opposite polarity fields observed well inside the penumbra in a sunspot 


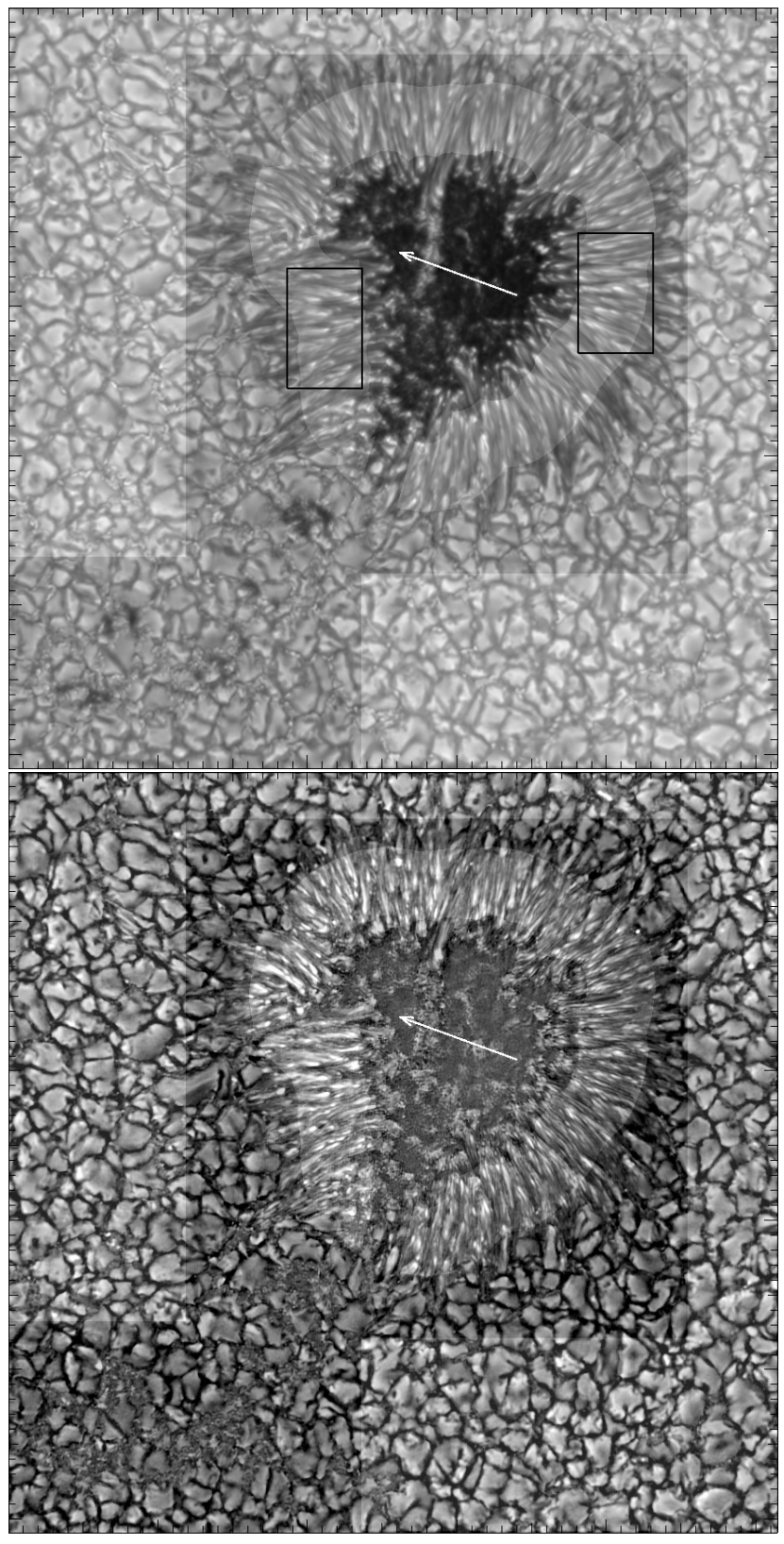

Fig. 1. Overview of the entire FOV observed. The highlighted contiguous area close to the boundary of the FOV corresponds to the area used as quiet Sun reference, the highlighted area in the penumbra corresponds to what is referred to as the "interior penumbra". The top panel corresponds to the $538 \mathrm{~nm}$ continuum, the right bottom to the COG velocity measured in the same line. Tick marks are at $1^{\prime \prime}$ intervals. The arrow points in the direction of Sun center. The dark rectangles outline sub-fields shown in Figs. 3 and 4.

located at small heliocentric distances (Ichimoto et al. 2007a; Franz 2011). However, Fig. 4 (upper row, mid panel) shows several small-scale patches where the LOS magnetic field is very weak at the center-side penumbra. The absence of opposite polarity patches in our LOS magnetic map may be related to the associated strong downward flows and the corresponding abnormal Stokes $V$ profiles that are not well captured with our simple (COG) method for estimating the LOS magnetic field. However, inspection of the Stokes $V$ images at $+26.3 \mathrm{pm}$ and $+30.7 \mathrm{pm}$ confirms that opposite polarity patches are found primarily in the outer penumbra and that only a few such patches are seen well inside the penumbra.

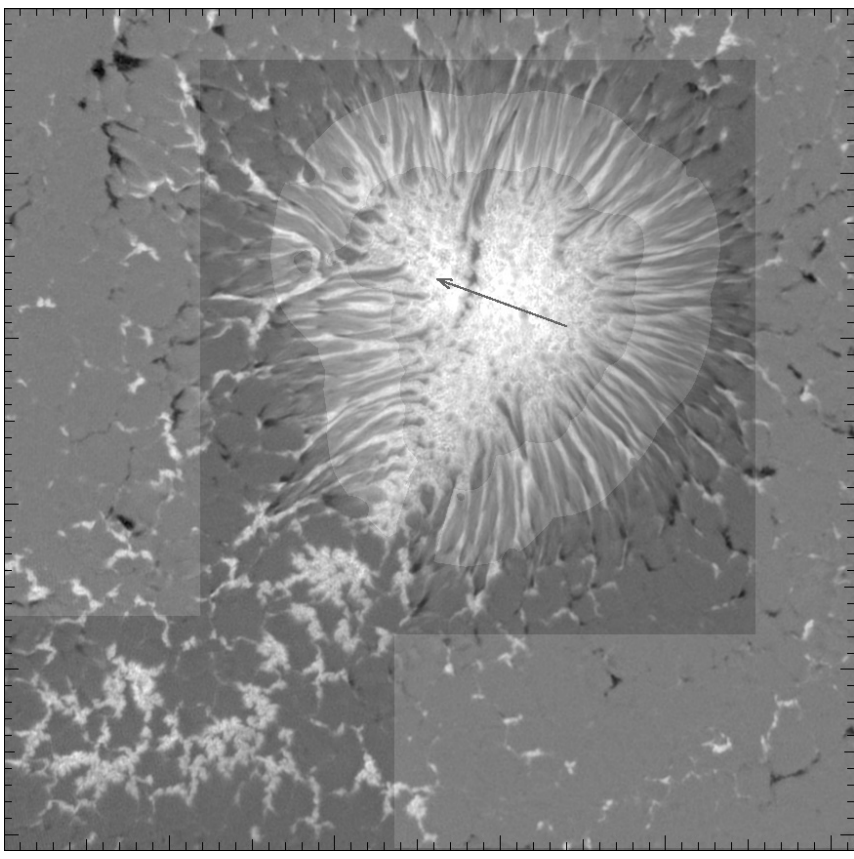

Fig. 2. The panel shows LOS component of the magnetic field over the same FOV as in Fig. 1. Tick marks are at 1 " intervals. The arrow points in the direction of Sun center.

\subsection{MOMFBD image reconstruction and alignment}

The images were corrected for gain and offset, using flats at a fixed continuum wavelength. The gain and dark corrected images from the 5380 and 6301/6302 lines and their corresponding broadband images were processed separately as two data sets with the Multi-Object Multi-frame Blind Deconvolution (MOMFBD) method (van Noort et al. 2005). In the MOMFBD image reconstruction process we conservatively compensated for only 36 Karhunen-Loeve aberrations. This leaves the small-scale wavefront errors uncompensated for at scales roughly $15 \mathrm{~cm}$ or smaller. With sufficiently good seeing, as in our case, the effect of such truncation is to leave residual straylight in the wings of the point-spread-function (PSF), whereas the core is well corrected, but with reduced peak strength (reduced Strehl) (Scharmer et al. 2010; Löfdahl $\&$ Scharmer 2012). We expect the restored images to have a spatial resolution close to the diffraction limit of 0.14 at $538 \mathrm{~nm}$ and 0.16 at $630 \mathrm{~nm}$.

After image reconstruction, the recorded Stokes images were demodulated for telescope polarization using the SST polarization model developed by Selbing (2005). Finally, the 6301 and and 5380 data were co-aligned by destretching the 6301 continuum image onto the 5380 continuum image over $16 \times 16$ subfields. The so-obtained matrix of geometrical distortions was then applied to all measured 6301 quantities. This process compensates to some extent also for any small effects of large scale flows and evolution of the structure during the $13 \mathrm{~s}$ time elapsed between the recording of the 5380 and 6301 data.

\subsection{Cavity error compensation and wavelength calibration}

In principle, it would be sufficient to measure the wavelength shifts from the cavity errors and to use these to correct the measured LOS velocities. However, straylight compensation of the science data also redistributes spatially the wavelength shifts from any cavity errors. Therefore, we first shifted the line profiles to a common wavelength scale by compensating for the 
cavity errors, and then applied the straylight compensation to the data.

The cavity error wavelength shifts were measured from the averaged flat-field images by fitting second-order polynomials to the core of the Stokes $I$ profile for each line. The wavelength shifts were determined from the coefficients of the fits. The broad 5380 line is well sampled (at 2.8 pm wavelength steps) and we used linear interpolation to shift the line profiles to a common wavelength scale and at the same time applying a first-order correction to compensate for the transmission profile of the CRISP pre-filters. The 6301 line has coarser wavelength sampling (4.4 pm) and we used cubic splines to resample the line profiles to $1.1 \mathrm{pm}$ steps before shifting the line profiles with linear interpolation according to the measured cavity errors. After compensation for cavity errors, we calculated average 5380 and 6301 line profiles from the high-lighted region surrounding most of the sunspot, shown in Figs. 1 and 2. We calibrated the wavelength scales such the line core velocities measured from the spatially averaged 5380 and 6301 line profiles correspond to $-917 \mathrm{~m} \mathrm{~s}^{-1}$ (Scharmer et al. 2011) resp. $-200 \mathrm{~m} \mathrm{~s}^{-1}$ (negative velocities correspond to blue-shifted profiles), in agreement with the convective blue-shifts obtained from simulations (de la Cruz Rodríguez et al. 2011). The 6301 convective blue-shift adopted is somewhat larger than the shift obtained from convection simulations $\left(-188 \mathrm{~m} \mathrm{~s}^{-1}\right)$ at disk center for the line core, when the averaged quiet Sun profile is convolved with the CRISP transmission profile (de la Cruz Rodríguez et al. 2011). As a check of this calibration, we calculated the average quiet Sun LOS velocity from the spatially resolved spectra and obtained $-130 \mathrm{~m} \mathrm{~s}^{-1}$ and $-220 \mathrm{~m} \mathrm{~s}^{-1}$ for the COG and line core 5380 velocities, and $150 \mathrm{~m} \mathrm{~s}^{-1}, 120 \mathrm{~m} \mathrm{~s}^{-1}$ and $-100 \mathrm{~m} \mathrm{~s}^{-1}$ for the $6301 \mathrm{COG}, 70 \%$ bisector and line core velocities. Averaging over the entire penumbra, the corresponding values are $-200 \mathrm{~m} \mathrm{~s}^{-1}$ and $-290 \mathrm{~m} \mathrm{~s}^{-1}$ for the 5380 line, and $50 \mathrm{~m} \mathrm{~s}^{-1}, 30 \mathrm{~m} \mathrm{~s}^{-1}$ and $-130 \mathrm{~m} \mathrm{~s}^{-1}$ for the 6301 line. The remaining convective blue-shifts in the 5380 line are similar to those expected from convection simulations (see SOM, Table S2) (see, http: //www. sciencemag. org/content/333/6040/316/suppl/DC1). The values for the 6301 line are reasonably consistent and indicate that the systematic errors are not larger than $\pm 150 \mathrm{~m} \mathrm{~s}^{-1}$ for this line. The weak blend in the red wing of the 6301 line most likely has a small influence on the velocities measured from the wings (the COG and $70 \%$ bisector measurements) of the 6301 line, but we made no attempt to compensate for that.

The sunspot is supposedly surrounded by a radially outward moat flow (e.g., Vargas Domínguez et al. 2008) of up to about $0.3-0.5 \mathrm{~km} \mathrm{~s}^{-1}$ that will have an influence on our wavelength calibration. No obvious evidence of such a flow can be seen in our Doppler maps, even with heavy spatial smearing of the individual Doppler maps. Since the region used for wavelength calibration surrounds most of the sunspot and because of the relatively small heliocentric distance, we expect the systematic errors in the wavelength calibration from the moat flow to be small.

\subsection{Straylight calibration and compensation}

Recently, the rms contrast of solar granulation was measured from Hinode data, combined with a detailed characterization of the straylight properties of the telescope and instrumentation used. Wedemeyer-Böhm (2008) determined the straylight PSF from images recorded during both a Mercury transit and a partial solar eclipse. After deconvolution of the granulation images for the measured stray-light PSF, the corrected granulation contrasts are close to those obtained from two independently developed
MHD simulation codes (the $\mathrm{CO}^{5} \mathrm{BOLD}$ and Stein-Nordlund codes; Wedemeyer-Böhm \& Rouppe van der Voort 2009). Danilovic et al. (2008) degraded synthetic continuum images at $630 \mathrm{~nm}$ calculated from simulations with the MURAM code with the PSF of Hinode. Taking into account only the PSF of a perfect unobscured $50 \mathrm{~cm}$ telescope, the rms contrast is reduced from $14.4 \%$ to $10.9 \%$. Including the effects of the large central obscuration and spider plus the CCD, reduces the rms contrast to $8.5 \%$. This is still higher than the observed value of $7 \%$. Danilovic et al. interpret this difference as the result primarily of small focus errors and/or other residual aberrations, but the work of Wedemeyer-Böhm \& Rouppe van der Voort (2009) as well as the Gaussian fits of Mathew et al. (2009) clearly indicate significant contributions also from the far wings of the PSF. We conclude that there are no longer scientific grounds for questioning the rms granulation contrast values obtained from numerical 3D simulations, and that the measured granulation contrast in highly resolved granulation images recorded with the SST and other solar telescopes therefore can be used to constrain the amount of straylight. We also note that the large central obscuration, the wide spider and aberrations and/or other imperfections combine to reduce the observed rms contrast of granulation to about $64 \%$ of that theoretically possible at wavelengths around $630 \mathrm{~nm}$ for a telescope with the diameter (but without the spider and central obscuration) of Hinode.

For the SST, the observed rms contrast is around $6.5-7 \%$ at $630 \mathrm{~nm}$ in the best "raw" images, but since the spatial resolution is higher than for Hinode, we could in principle reach $14 \%$ contrast with a perfect SST operating outside the Earth's atmosphere. MOMFBD image reconstruction increases the rms contrast somewhat to about $7.5 \%$ at $630 \mathrm{~nm}$ and $9 \%$ at $538 \mathrm{~nm}$ (Scharmer et al. 2010). Clearly, the SST data must contain on the order of $50 \%$ straylight, operating at scales corresponding to granulation or larger. At the same time, "conventional" straylight, often characterized by a PSF having very wide (e.g., Lorentzian) wings, must be low in this and other SST data: the minimum umbral intensity in the sunspot presently discussed is $15 \%$ at $538 \mathrm{~nm}$ and $18 \%$ at $630 \mathrm{~nm}$. Measurements of straylight made during the 2004 Venus transit suggests on the order of $4 \%$ at $390 \mathrm{~nm}$ and $9 \%$ at $700 \mathrm{~nm}$ (Kiselman 2008). Measurements of straylight from the primary focal plane just outside the SST focal plane and including all relay optics as well as CRISP and the CCD itself suggests levels of "conventional" straylight of at most a few percent (Löfdahl \& Scharmer 2012). The origin of the dominant source of straylight is largely unknown but is now suspected to be mostly from aberrations, including the high-order aberrations truncated in MOMFBD processing, high-altitude seeing and/or effects from the relatively long integration times (17 ms) used (Scharmer et al. 2010; Löfdahl \& Scharmer 2012). This conclusion receives at least partial support from a recent comparison between speckle and MOMFBD image restorations of spectropolarimetric data recorded at the German VTT. This comparison demonstrated that speckle restorations and MFBD processing (identical to MOMFBD processing, but with a single wavelength channel) gives significantly higher granulation contrast than with MOMFBD (Puschmann \& Beck 2011).

An important component of our data processing is therefore straylight compensation. In the Supplementary Online Material (hereafter SOM) of Scharmer et al. (2011), we discuss this in detail for the 5380 line, here we summarize the analysis made. We used snapshots from field-free simulations obtained with the Stein \& Nordlund (1998) code, degraded with the PSF of a perfect 1-m solar telescope. This constitutes our "true" theoretical data. We assumed that the actual PSF of the MOMFBD restored 
science data corresponds to a diffraction limited core with reduced peak strength (reduced Strehl) and enhanced wings due to straylight as follows

$I_{\mathrm{o}}=(1-\alpha) I_{\mathrm{t}}+\alpha I_{\mathrm{t}} * S(W)$

where $I_{\mathrm{o}}$ and $I_{\mathrm{t}}$ are the observed and "true" intensities at any wavelength and polarization state, $\alpha$ the straylight fraction, "*” denotes convolution and $S$ is the straylight PSF, having a full width at half maximum (FWHM) $W$. This corresponds to a total PSF $P$ of

$P=(1-\alpha) \delta+\alpha S(W)$,

where $\delta$ is Dirac delta function. Note that this PSF corresponds to assuming that the core of the PSF is perfectly compensated for by MOMFBD processing; this is consistent with our approach of first degrading the synthetic simulation data with the diffraction limited PSF of the SST. When using Eq. (2) to deconvolve the data we thus compensate only for straylight. We used this PSF to both degrade the simulated data and to deconvolve the observed data. As basis for our comparison, we calculated both synthetic continuum images and line profiles corresponding to a heliocentric distance of $15^{\circ}$. We then calculated 5380 line core LOS velocities from both the synthetic and the observed data. The calculated quantities compared were the rms continuum intensity, the 5380 line core rms velocity and the spatially averaged line core velocity ${ }^{4}$. This comparison does not give much guidance about the shape of the straylight PSF. However, the low umbra intensity observed implies that the straylight PSF cannot be very wide. In particular, a PSF having extended Lorentzian wings would lead to observed umbra intensities on the order of $50 \%$, given that the level of straylight must be on the order of $50 \%$ to explain the observed granulation contrast. If we assume that the true minimum umbra intensity is not less than half of the measured values, then up to $7-9 \%$ of the straylight could be from a Lorentzian PSF. A similar problem with the umbra intensity arises even with a Gaussian PSF, if it is too wide. Based on extensive tests, we conclude that the majority of the straylight must be contained inside a PSF with a FWHM that is less than about $2^{\prime \prime}$, else the restored umbra intensity is close to zero or negative (Scharmer et al. 2011).

Our best agreement with granulation data, taking into account the constraints from the umbra intensity, is a Gaussian PSF with a FWHM of about only 1'.2 and a straylight contribution of $\alpha=58 \%$ at $538 \mathrm{~nm}^{5}$. We have adopted these values for the 5380 line. We use the same FWHM for the 6301 line, but with lower straylight contribution, $\alpha=50 \%$ at $630 \mathrm{~nm}$, since our present observations as well as those discussed by WedemeyerBöhm \& Rouppe van der Voort (2009) suggest that the SST straylight increases at shorter wavelengths. This leads to a quiet

\footnotetext{
${ }^{4}$ The $C_{\text {I }} 5380$ line is very temperature sensitive and weakens considerably in dark granular and penumbral lanes. This makes it very sensitive to straylight in dark (cool) lanes, explaining the large convective blueshift $\left(-917 \mathrm{~m} \mathrm{~s}^{-1}\right.$ at $15^{\circ}$ heliocentric distance). This also makes the spatially averaged 5380 velocity from spatially resolved line profiles a sensitive indicator of straylight. Indeed, even the straylight compensated SST data leaves a residual convective blueshift of about $-240 \mathrm{~m} \mathrm{~s}^{-1}$ when averaging LOS velocities from the spatially resolved line profiles for quiet Sun (this residual is consistent with what is obtained from simulations). A similar blue-shift is obtained when averaging the 5380 line core velocities over the penumbra.

5 A narrow straylight PSF is consistent with main contributions from high-order aberrations, such as from seeing or possibly from the adaptive mirror (Scharmer et al. 2010; Löfdahl \& Scharmer 2012).
}

Sun rms granulation contrast of about $13.3 \%$ at $630 \mathrm{~nm}$, which is somewhat lower than from simulations (Danilovic et al. 2008; Scharmer et al. 2010), but the "quiet" region we observed does contain some small fraction of strong field that is likely to reduce the overall contrast. Admittedly, this determination of the straylight PSF is not precise, but the main effect of the straylight is present already when its PSF includes about one granule. Compensation with a wider straylight PSF will require somewhat smaller values of $\alpha$, but the overall results do not change qualitatively. Whereas the accuracy of our measured intensities and LOS velocities thus is limited by the uncertainties about the precise shape of the straylight PSF, we are confident that the conclusions drawn about the clear existence of correlations between measured continuum intensities and LOS velocities, as well as about the existence of dark convective downflows in the interior penumbra, are robust.

\subsection{LOS velocities and magnetic field measurements}

When measuring spectral line properties at low temperatures (low continuum intensities) there is always a concern about the possible influence of blends from (mostly) molecular lines. The 5380 line weakens considerably at reduced temperatures and must disappear in the umbra, strongly aggravating any such influence of blends. In SOM, we established that the influence of blends is small near the core of the 5380 line at continuum intensities corresponding to granulation and penumbral filaments. However, there is evidence of weak blends both in the far blue and red wings (see Fig. S5 in SOM) in the penumbral dark structures and in the dark intergranular lanes. These blends are obvious in the umbra (where also a strong molecular blend, shifted by about 3-3.5 pm to the blue of the $\mathrm{C}_{\mathrm{I}}$ line, is present - see $\mathrm{SOM}^{6}$ ). To mitigate the influence of the blends in the wings, we initially choose to estimate LOS velocities from the line core only, by fitting a second-order polynomial to 5 wavelengths closest to the line minimum (Scharmer et al. 2011). In the present work, we estimate also 5380 COG velocities, expected to be dominated by flows even closer to the photosphere than from the line core. In order to remove influence from the above mentioned blue/red blends, we crop the 5380 line profiles by excluding the first 6 and last 2 wavelengths shown in Fig. S3 in SOM. This leads to underestimates of the strengths of the strongest blue- and redshifted COG velocities, as is the case when the COG method is applied to LOS magnetic field measurements (Cauzzi et al. 1993), but these data nevertheless add support to the earlier line core measurements and also provide interesting information about flows in the deepest observable layers of the penumbra.

We measured the 6301 line core shifts from the 3 wavelengths nearest to the line minimum, the COG velocity from the entire line profile and (using the spline interpolated line profiles) the $70 \%$ bisector velocity, where the $70 \%$ intensity level is relative to the line minimum intensity at each pixel. We estimated the LOS magnetic field $B_{\text {LOS }}$ by applying the COG method to Stokes $I+V$ and $I-V$ (Rees \& Semel 1979; Cauzzi et al. 1993; Orozco Suárez et al. 2010) and from the difference in the two COG wavelengths $\delta \lambda$ we obtained $B_{\mathrm{LOS}}=$ $\delta \lambda\left(9.33410^{-13} \lambda^{2} g_{\mathrm{L}}\right)^{-1}$, where wavelengths are given in units of Angstrom, and $g_{\mathrm{L}}$ is the Landé splitting (=1.667 for the 6301 line).

\footnotetext{
${ }^{6}$ This blend has now been identified to be from $\mathrm{Mg} \mathrm{H}$ by Uitenbroek, Dumont and Tritschler (in prep.).
} 


\subsection{High-pass spatial filtering and masks}

In order to investigate relations between small-scale fluctuations in intensity, LOS velocity and magnetic field, we make repeated use of high-pass filtered (unsharp masked) quantities. In particular, such filtering strongly reduces radial variations of the intensity in the penumbra, large-scale radial and azimuthal variations in the LOS component velocities from the horizontal (Evershed) flow and the horizontal component of the magnetic field. For example, the high-pass filtered continuum intensity $\delta I_{\mathrm{c}}$ is calculated as

$\delta I_{\mathrm{c}}=I_{\mathrm{c}}-G\left(W_{2}\right) * I_{\mathrm{c}}$

where $G$ is a Gaussian profile having a FWHM width $W_{2}$ of 20 pixels, or approximately 1 '. 2 . The same high-pass filter is used with the measured LOS velocities and LOS magnetic field. The FWHM used here is coincidentally the same as used for the straylight compensation, but the precise value of the FWHM is not critical for the analysis. It might be argued that a filter kernel with a larger FWHM would be more appropriate, but we prefer a narrow filter in order to reduce the spatial mixing of information across the borders to the umbra and the surrounding quiet Sun.

An obvious effect of the spatial filtering is that the absolute zero point of any filtered quantity is lost and that all filtered quantities are referenced only to a local mean, defined by the filter kernel. In the following, we therefore analyze both the filtered velocities to show their relation to local intensity fluctuations, and the absolute (unfiltered) velocities to demonstrate that the darkest penumbral structures are indeed associated with downflows.

In Fig. 5 are shown in the top row the $538 \mathrm{~nm}$ continuum image, the 5380 COG velocity and the LOS component of the magnetic field. In the bottom row are shown the same quantities after applying the previously defined high-pass filter. Clearly, the high-pass filter strongly reduces both systematic radial variations as well as differences between the limb and center sides of the penumbra, but leaves the small-scale structures intact.

For some of our fits (see Sect. 2.6), we use the spatially filtered quantities to define spatial masks, isolating certain measured parameters in a given range. In particular, we use the filtered $538 \mathrm{~nm}$ continuum image to define masks that have intensities in a specific range to estimate the relation between intensity and vertical and radial flows (see Sects. 3.1 and 3.2). We also use the filtered LOS magnetic field map to identify penumbral structure where the LOS magnetic field is locally strong (spines) or weak and nearly horizontal (inter-spines). These masks, shown in Fig. 6, are based on the high-pass filtered $B_{\mathrm{LOS}}$ map, with thresholds set such that roughly $33 \%$ of the penumbra is contained in each of the two masks.

\subsection{Azimuthal fits}

The sunspot observed is at $15^{\circ}$ heliocentric distance, such that the measured LOS velocity contains a mixture of contributions from both vertical and (strong) radial flows ${ }^{7}$. We must resort to statistical methods to separately determine average properties of the radial and vertical flows. The presently observed penumbra has boundaries to the umbra and quiet Sun that are rather

\footnotetext{
7 A sunspot located precisely at disk center would be allow clean measurements of vertical velocities. However, even a few degrees heliocentric distance leads to significant contributions from radial Evershed flows that nearly always must be accounted for in such measurements, as demonstrated by Bharti et al. (2011).
}

irregular, but the penumbral filaments and the radial extent of the penumbra appear similar on the disk center and limb sides. We assume that the small-scale velocity field and magnetic field fluctuations do not vary in amplitude with azimuth angle in order to separate the observed LOS component of the velocity into its radial and vertical components.

Ignoring any azimuthal components of the flow field, the relation between the LOS velocity $v_{\text {LOS }}$, the radial (horizontal) velocity $v_{r}$, the vertical velocity $v_{z}$, the heliocentric distance $\theta$ $\left(=15^{\circ}\right)$ and the azimuthal angle $\phi$ is then ${ }^{8}$

$v_{\mathrm{LOS}}=-v_{r} \cos \phi \sin \theta+v_{z} \cos \theta$

(Plaskett 1952). Fits based on this relation have been used to disentangle the radial and vertical components of penumbral velocity and/or magnetic fields from LOS measurements by numerous earlier investigators (e.g., Maltby 1964; Title et al. 1993; Schlichenmaier \& Schmidt 2000; Bellot Rubio et al. 2003; Tritschler et al. 2004; Langhans et al. 2005; Bellot Rubio et al. 2006; Sánchez Almeida et al. 2007; Scharmer et al. 2011). The sign conventions here are that $\phi$ is zero in the disk center direction, radial outflows are counted positive, and vertical velocities as well as velocities in the direction toward the observer are counted as negative. Our procedure for defining the azimuth angle and radial distances within the penumbra is described in SOM. The zero-point azimuth direction was determined from full disk images recorded by the Solar Dynamics Observatory (SDO) close to the time of our observations and is indicated by the arrows shown in, e.g., Figs. 1 and 2. Guided by the orientation of the penumbral filaments, we determined an approximate center of the sunspot from which straight lines line up as well as possible with the penumbral filaments. The umbra/penumbra boundary was identified by applying an intensity threshold and removing the light bridge and innermost part of a few filaments. From that boundary six radial zones were defined by successively applying a dilate operation using a circular kernel of $1.5^{\prime \prime}$ radius. The boundaries of the six radial zones are shown as dark contours in Fig. 5. Examples of azimuthal fits made at different high-pass filtered $538 \mathrm{~nm}$ continuum intensities can be found in Fig. 7. These show fundamental differences between the spines and inter-spines: in the inter-spines the amplitude of the azimuthal variation of $v_{\text {LOS }}$ implies a strong radial component of the LOS velocity that is much weaker in the spines and virtually absent at low continuum intensities.

\section{Results}

Figures 3 and 4 show all measured quantities within two $5^{\prime \prime} \times$ $8^{\prime \prime}$ subfields on (mostly) the limb and disk center sides, with tick marks separated at $1^{\prime \prime}$ intervals. The first and last panels in the top row show the $538 \mathrm{~nm}$ and $630 \mathrm{~nm}$ continuum images. These appear nearly identical but the continuum image at $538 \mathrm{~nm}$ shows somewhat higher spatial resolution, as expected. The first two panels in the bottom row show the 5380 COG and line core velocities. These are quite similar, but with significant differences for the smallest flow features shown. Although not so obvious in Fig. 4, there is an overall tendency, also outside the sunspot, for the COG velocity map to show stronger smallscale flows than for the 5380 line core velocity map. This is interpreted to mean that the flows with the smallest scales change rapidly with height, whereas the large-scale flows show weaker gradients with height.

8 The same relation, but with different sign conventions, is used to resolve $B_{\mathrm{LOS}}$ into its radial and vertical components. 

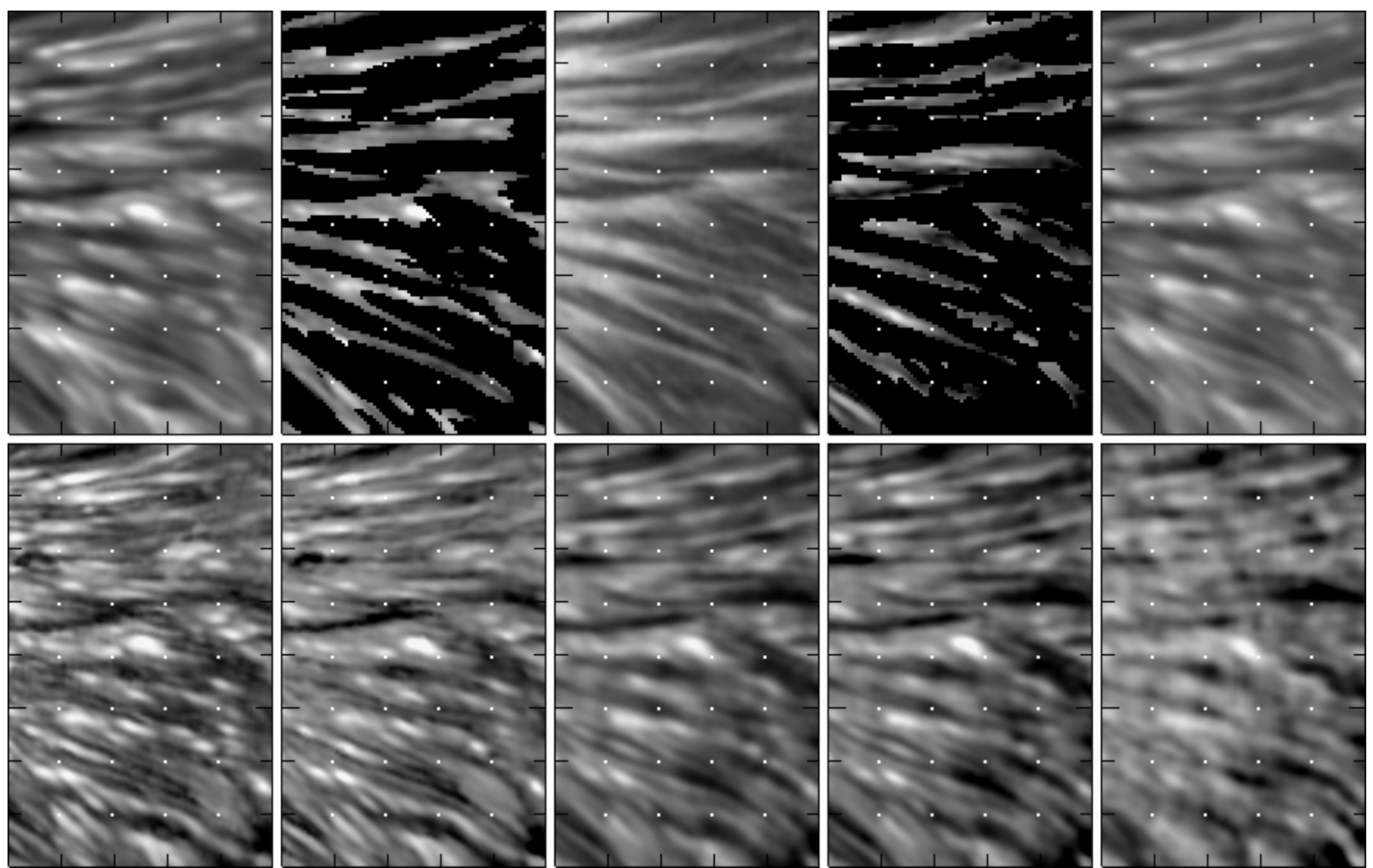

Fig. 3. The figure shows a $5^{\prime \prime} \times 8^{\prime \prime}$ sub-field from the right-hand side (mostly limb-side) penumbra, outlined in Fig. 1 . The top row shows the $538 \mathrm{~nm}$ continuum, the 5380 COG velocity within the spine mask, the LOS magnetic field, the 5380 COG velocity within the inter-spine mask, and the 6301 continuum intensity. The bottom row shows the 5380 COG velocity, the 5380 line core velocity, the 6301 COG velocity, $630170 \%$ bisector velocity and the 6301 line core velocity. The velocity maps have been scaled individually to enhance fine structure. Maps shown represent original quantities and have not been processed with unsharp masking. Tick marks and small white dots are at $1^{\prime \prime}$ intervals.

The 6301 flow maps in general show much less fine structure than the 5380 flow maps. This is not a question of spatial resolution, as the continuum images demonstrate, but most likely is due to either or both of two effects: the first is that smallscale upflows do not extend to the same height as large-scale strong upflows. The second effect is the relatively large formation height range of the 6301 line, aggravated by the limited spectral resolution of CRISP, causing smearing of flow topologies that change with height within the formation range. We note that the 6301 line core velocity (last panel) is the most "fuzzy" of these flow maps on the limb side, but not on the disk center side. We also note the $6301 \mathrm{COG}$ and $70 \%$ bisector flow maps (third and fourth panels in the bottom row of Figs. 3 and 4) appear almost identical. In the following, we do not discuss these flow maps separately. We attribute this similarity to relatively low spectral resolution of CRISP, smearing out the height variations of the flow field.

We emphasize the small azimuthal scale of almost all the flow structures, in particular the (dark) red-shifted lanes in between the (bright) blue-shifted features. Many of these flow structures appear to have azimuthal scales close to the SST diffraction limit $\left(0\right.$ ' $^{\prime} 14$ at $\left.538 \mathrm{~nm}\right)$. This clearly illustrates the difficulties of earlier (failed) attempts aimed at detecting convective downflows (i.e., that dark features are associated with downflows) in the interior penumbra at much lower spatial resolution (Bellot Rubio et al. 2010; Franz \& Schlichenmaier 2009; Franz 2011), as demonstrated recently also from analysis of synthetic spectra calculated from simulation data and degraded to the spatial resolution of SST (Bharti et al. 2011). In the following, we characterize the complicated relations between the observed small-scale variations in the continuum intensity, flow field and magnetic field, and attempt to explain some of the observed differences between the limb and disk center sides of the penumbra.

Finally, we note that the LOS magnetic field (mid panel, upper row in Figs. 3 and 4), shows only weak evidence for variations that can easily be linked to the small-scale velocity field. However, the LOS magnetic field in Fig. 4 shows several narrow dark structures, where either the polarity reverses or where the LOS magnetic field is nearly zero (according to the present COG measurements). These can in most cases be linked to locally red-shifted flow features. We will discuss these features in more detail in a forth-coming paper.

\subsection{Intensity and LOS velocity correlations}

A quick glance at Figs. 3 and 4 makes it evident that the measured small-scale LOS velocities are strongly related to the continuum intensity: e.g., blue-shifted (shown bright) features can in almost all cases be associated with a locally bright continuum feature. This does not necessarily mean that there exist simple one-to-one relations between these quantities: it is easy to find examples where a relatively strong blue-shifted feature shows only a marginal brightening in the continuum 
G. B. Scharmer and V. M. J. Henriques: SST/CRISP observations of convective flows in a sunspot penumbra
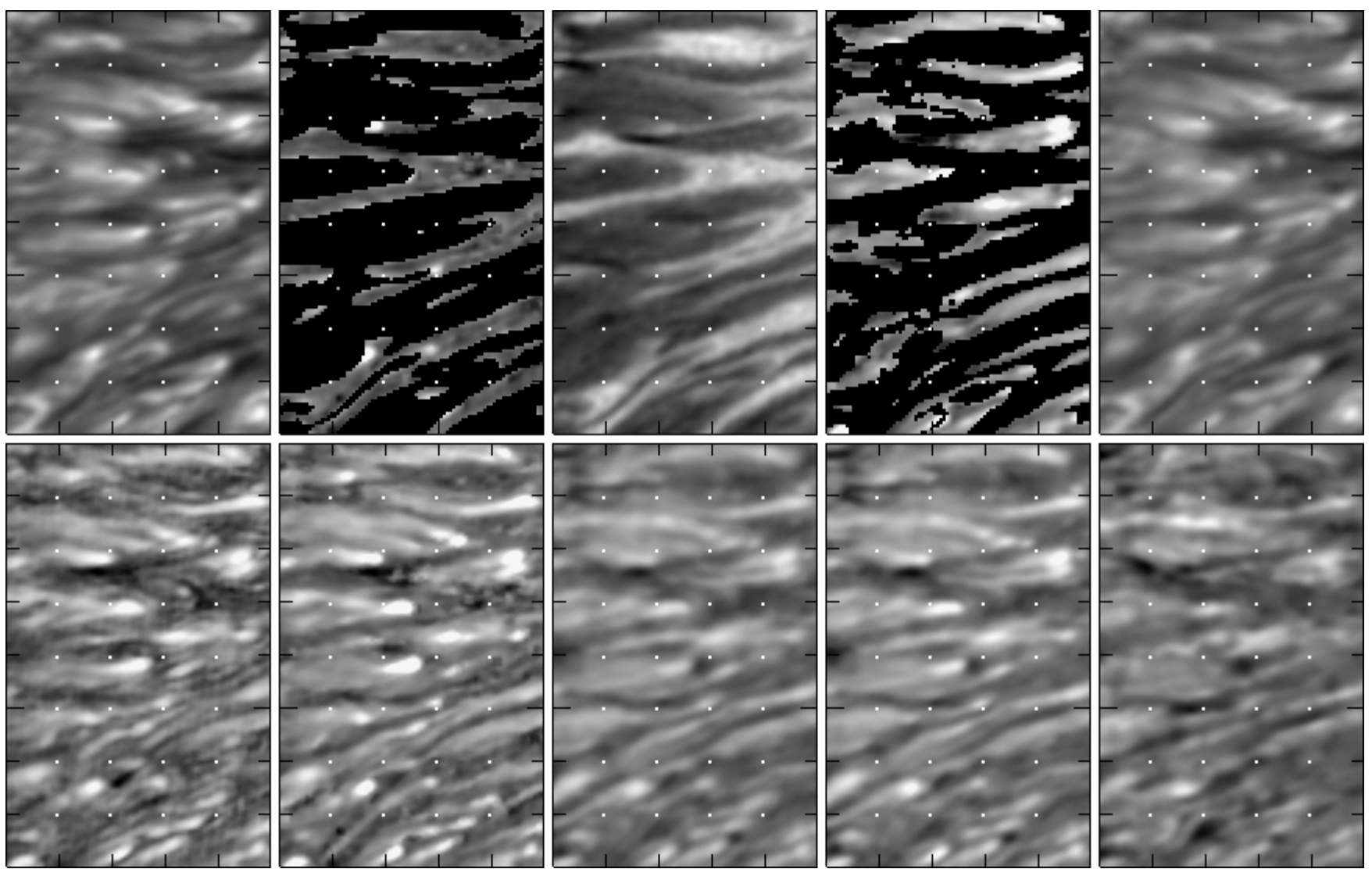

Fig. 4. The panel shows a $5^{\prime \prime} \times 8^{\prime \prime}$ sub-field from the left-hand side (mostly disk center-side) penumbra outlined in Fig. 1. The arrangement of the panels is the same as shown in Fig. 3.

and vice-versa. In other cases, the flow features and continuum structures appear somewhat displaced spatially relative to each other. There is no reason to expect a perfect correlation between intensity (temperature) and flow velocities: even for quiet Sun granulation, this correlation is less than about $80 \%$. We first quantify these correlations in the simplest possible manner. Figure 8 shows the correlation between the high-pass filtered $538 \mathrm{~nm}$ continuum intensity and the high-pass filtered LOS velocity for the interior penumbra (top row) and quiet Sun (bottom row). As shown in Fig. 5, the main effect of high-pass filtering the LOS velocity map is to remove the systematic differences in LOS velocity between the disk center and limb sides - these differences are from the radial (horizontal) flow.

After high-pass filtering the LOS velocity map, the remaining small-scale flow field structures appear similar on the disk center and limb sides, suggesting a dominance of vertical flows (cf., Beckers \& Schröter 1969, for similar arguments). The correlations between intensity and LOS velocity in Fig. 8 are obvious, the corresponding correlation coefficients are given in Table 1 and range from $-60 \%$ to $-69 \%$ (except in the 6301 line core, where it is $-40 \%$ ) for the penumbra. For the quiet Sun, the corresponding correlations are stronger and range from $-73 \%$ to $-80 \%$ ( $-59 \%$ at the 6301 line core). The values for the quiet Sun are similar to those found from bisector measurements close to the continuum in the 6301 line by Franz (2011), -78\%, the lower values found by him for the penumbra are likely to be explainable by the much lower spatial resolution of the Hinode data and the different methods of analysis used by Franz and us. Irrespective of these differences, our conclusion about the existence of strong relations between the continuum intensity and the flow field does not rely on the precise values of the correlation coefficients found; as stated earlier they are obvious in Figs. 3 and 4 . The correlation coefficients calculated here only quantify this relation in a simple way that is easily testable against simulations. In the following we will analyze this in more detail, using an independent technique. We finally note (Table 1) that the rms velocities obtained for the penumbra are on the order of $65-70 \%$ of those for the quiet Sun, such that they suffice to explain the penumbral heat flux (cf., Scharmer et al. 2011, for a more detailed discussion).

\subsection{LOS velocity and magnetic field correlations}

Figure 9 shows the correlation between the measured LOS magnetic field and LOS velocities in the interior penumbra, measured with the COG method and from the line core of the 5380 line, and the $70 \%$ bisector and from line core of the 6301 line. Note that the quantities shown in this figure have not been spatially filtered. All correlations shown (and that for the 6301 COG velocity) have a roughly triangular shape with the "corners" characterized as follows: the strongest blueshifts corresponds to weak ( $\sim 400 \mathrm{G})$ LOS magnetic field with the same polarity as the leading polarity of the spot. The strongest redshifts also correspond to weak LOS magnetic fields but with a polarity that is opposite to that of the spot. Almost all this opposite polarity field is in the limb-side penumbra, but a few such patches can also be found in the center-side penumbra. Finally, the strongest LOS magnetic field (up to about $1600 \mathrm{G}$ ) systematically corresponds to small blue- and redshifts.

In the lower-right panel of Fig. 10 we show the variation of the radial velocity with the strength of the high-pass filtered 

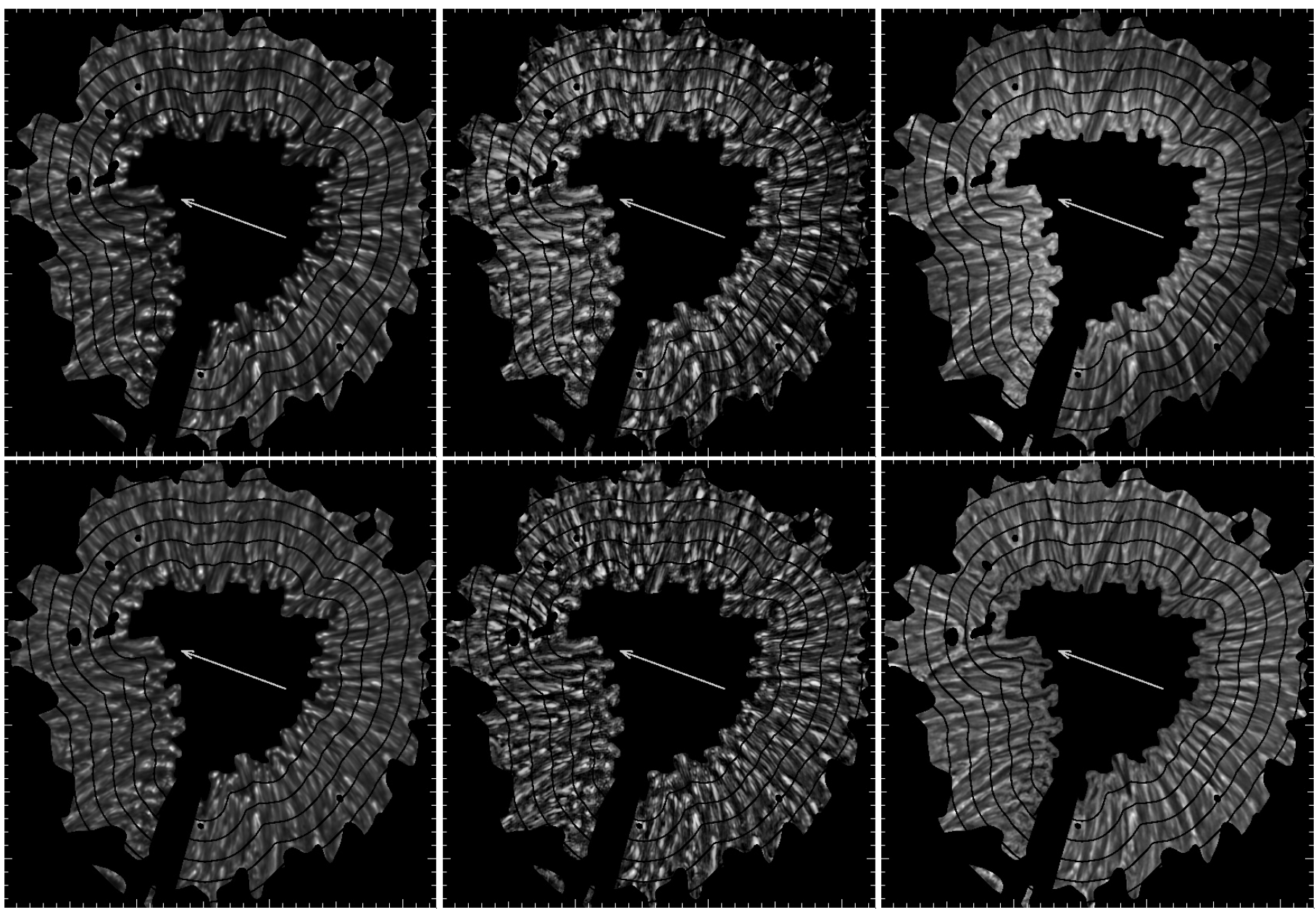

Fig. 5. Examples of measured quantities with superimposed contours outlining the six radial zones in the penumbra, numbered 1 (adjacent to the umbra) to 6 (the outermost penumbra). Radial zones 2-4 constitute what is referred to as the "interior penumbra". In the top row are shown (left to right) the $538 \mathrm{~nm}$ continuum intensity, the COG velocity in the same line and the LOS component of the magnetic field, obtained with the COG method. In the lower row are shown the same quantities but high-pass spatially filtered to remove large-scale azimuthal and radial variations. Tick marks are at 1 " intervals. The arrow points in the direction of Sun center.

LOS component of the magnetic field. This plot was obtained by creating LOS magnetic masks as explained in Sect. 2.5 and making azimuthal fits of the measured LOS velocities (Sect. 2.6) to separately determine the vertical and radial velocities. This plot shows a dramatic variation of the radial velocity with the filtered LOS magnetic field: where $B_{\mathrm{LOS}}$ is locally very weak, the radial velocity reaches up to $5 \mathrm{~km} \mathrm{~s}^{-1}$ at the height of formation of the 5380 line, where it is very strong the radial velocity drops to less than $0.5 \mathrm{~km} \mathrm{~s}^{-1}$ ! At heights corresponding to the formation of the 6301 line, the radial velocities are systematically lower, but the overall trends are the same as for the 5380 line. The lower-left plot in Fig. 10 shows that the strength of the spatially filtered LOS magnetic field is closely related to the inclination of the magnetic field: where this quantity is small, the magnetic field is nearly horizontal and where it is strong, the magnetic field is inclined by about $50^{\circ}$ to the vertical. Assuming that most of the variation in the (unfiltered) LOS magnetic field comes from inclination changes, rather than from field strength variations, these plots explain the triangular shape of the correlations shown in Fig. 9. Where the LOS magnetic field is weak (nearly horizontal field), strong radial flows produce considerable spread in the measured LOS velocities, with the extremes corresponding to the lower-left and lower right "corners" of the triangles. Where the LOS magnetic field is very strong, corresponding to more vertical field (upper "corner"), radial flows are virtually absent, such that the spread in the measured LOS velocities is limited to that of the (convective) vertical flows.

In the remaining panels of Fig. 10 we show the results of fits of $B_{\mathrm{LOS}}$ to the high-pass filtered $B_{\mathrm{LOS}}$ map. These plots show that selecting locally very weak LOS magnetic field corresponds to selecting a component of the penumbral field that is relatively weak (field strength of about $850 \mathrm{G}$ ) and (not surprisingly) almost horizontal (inclination approximately $80^{\circ}$ ). Selecting the locally strongest LOS magnetic field corresponds to selecting the part of the magnetic field that is stronger (about $1200 \mathrm{G}$ ) and more vertical (inclination approximately $50^{\circ}$ ). These properties agree with those of spines and inter-spines (Lites et al. 1993), although the much lower spatial resolution of their data compared to ours means that it is not obvious that we are identifying the same penumbral structures. Nevertheless, we can establish that for the observed sunspot, the strong horizontal (Evershed) flow prevails in the inter-spines and is virtually absent in the spines. This division of the penumbra into two main components with quite different magnetic and flow properties is consistent with numerous results obtained from inversions based on spectropolarimetric data at much lower spatial resolution than here (Solanki \& Montavon 1993; Martínez Pillet 2000; Schlichenmaier \& Collados 2002; Bellot Rubio et al. 2003, 2004; Borrero et al. 2007; Tritschler et al. 2007), and is at the center of interpretations in terms of a "flux tube" (at a smaller 
G. B. Scharmer and V. M. J. Henriques: SST/CRISP observations of convective flows in a sunspot penumbra

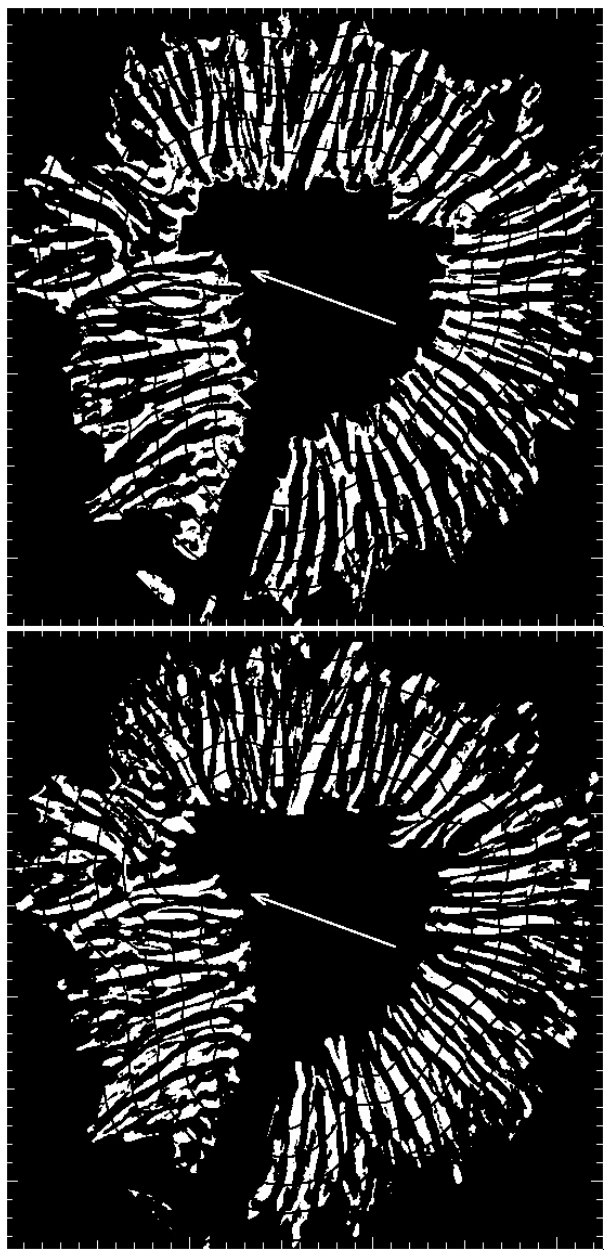

Fig. 6. Spine (upper panel) and inter-spine (lower panel) masks, obtained by thresholding the spatially filtered LOS magnetic field map, shown in the lower-right panel of Fig. 5. The thresholds have been set such that roughly $33 \%$ of the penumbra is contained in each of the two masks.

scale than our spines) and a "background component". The existence of these two fairly distinct populations is clearly illustrated in Fig. 11, showing the 5380 COG and line core and the 6301 COG LOS velocity field within the inter-spine mask (top row) and within the spine-mask (bottom row). In the top row (inter-spines), we see clearly the signatures of the Evershed flow: strong blue-shifts on the disk center side and strong red-shifts on the limb side. In the spines, these signatures are missing entirely, showing that the Evershed flow is virtually absent.

Figure 12 shows the radial variation of the average properties determined for spines and inter-spines, indicating a magnetic field that dips down (inclination larger than $90^{\circ}$ ) in the inter-spines in the outer part of penumbra, consistent with earlier findings. In comparison, the plot of the average vertical velocity for the inter-spines shows a cross-over to positive velocities (downflows) further toward the center of the sunspot, clearly indicating that the average magnetic field and flow field are not precisely aligned (a similar result was obtained from the 5380 Stokes $V$ and LOS velocities, see SOM Fig. S10, lowerleft panel). Systematic vertical and radial flows are small in the spines, but the inter-spines show strong radial flows up to $5 \mathrm{~km} \mathrm{~s}^{-1}$ in the interior penumbra and systematic upflows in the inner and downflows in the outer penumbra. Ignoring the existence of the small-scale intensity correlated vertical (convective)
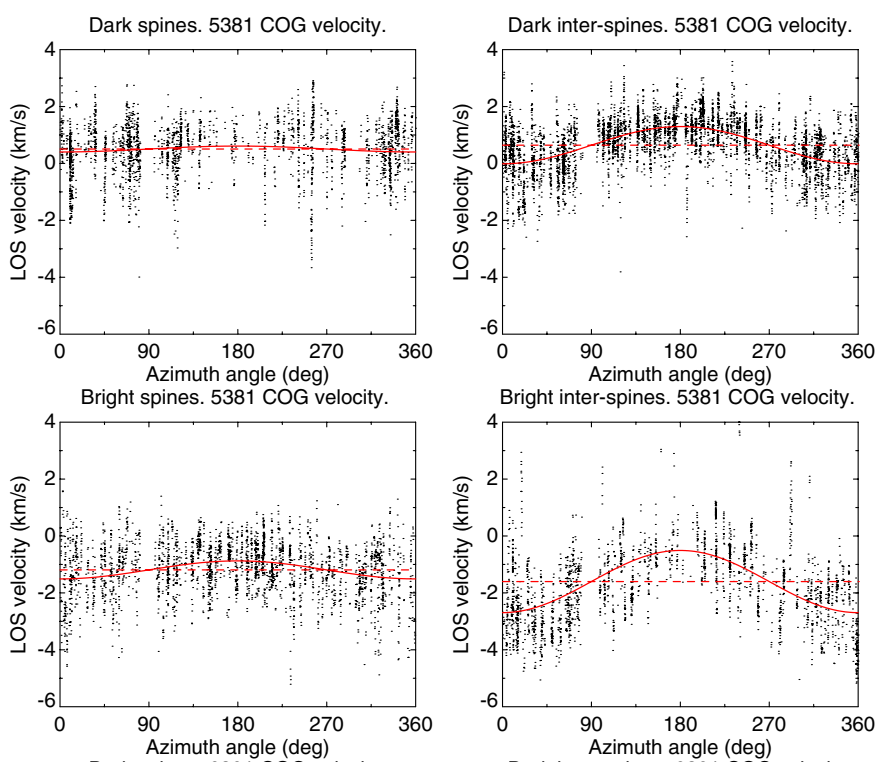

Bright inter-spines. 5381 COG velocity.
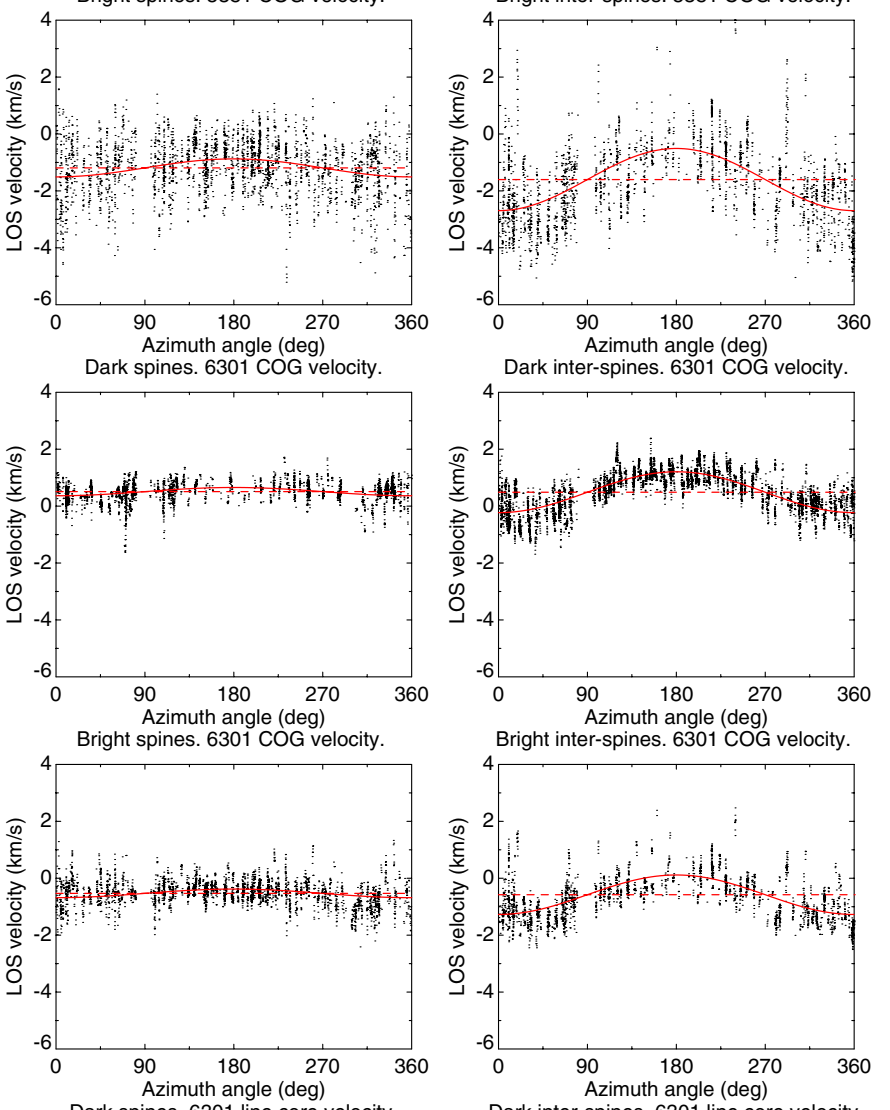

Dark spines. 6301 line core velocity.

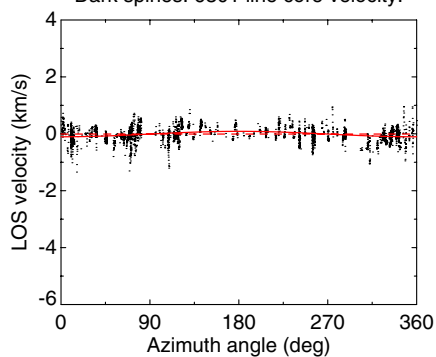

Dark inter-spines. 6301 line core velocity.
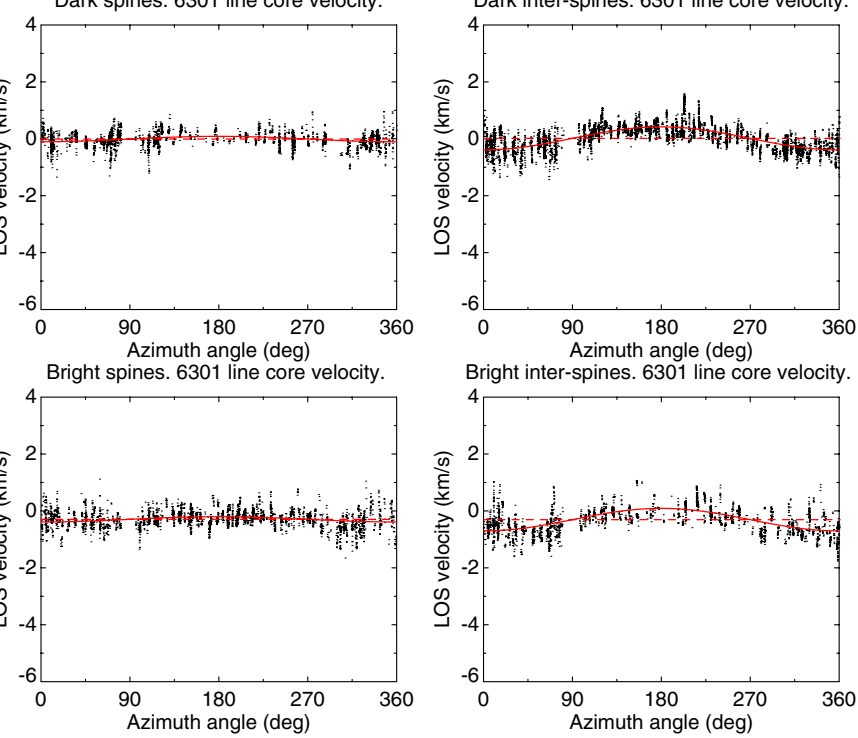

Fig. 7. Examples of azimuthal fits of the absolute (unfiltered) LOS velocities to Eq. (4), measured with different methods in the 5380 and 6301 lines, for relatively dark $\left(\delta I_{\mathrm{c}} \approx-0.19\right)$ and bright $\left(\delta I_{\mathrm{c}} \approx 0.19\right)$ structures. The left column shows the data (dark dots) and fits (red curves) for spines, the right column for inter-spines. 

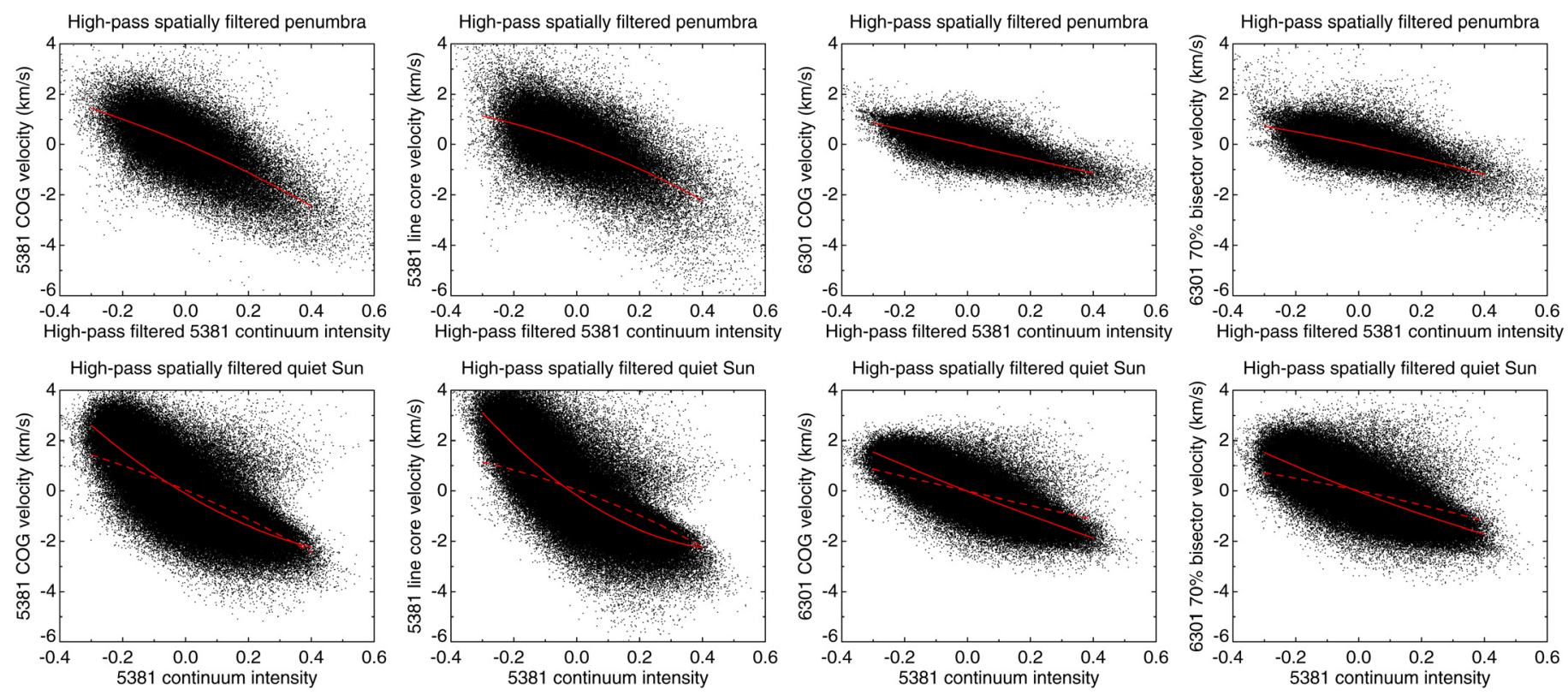

Fig. 8. Correlation between the high-pass filtered $538 \mathrm{~nm}$ continuum intensity and the high-pass filtered LOS velocity, measured with different methods in the 5380 and 6301 lines. The top row shows plots for the "interior penumbra", the bottom row corresponding plots for quiet Sun. For easier comparison, the red curves show in the upper panels are shown as dashed curves in the lower panels. Note that the velocities plotted are referenced to local averages by spatial filtering (unsharp masking).
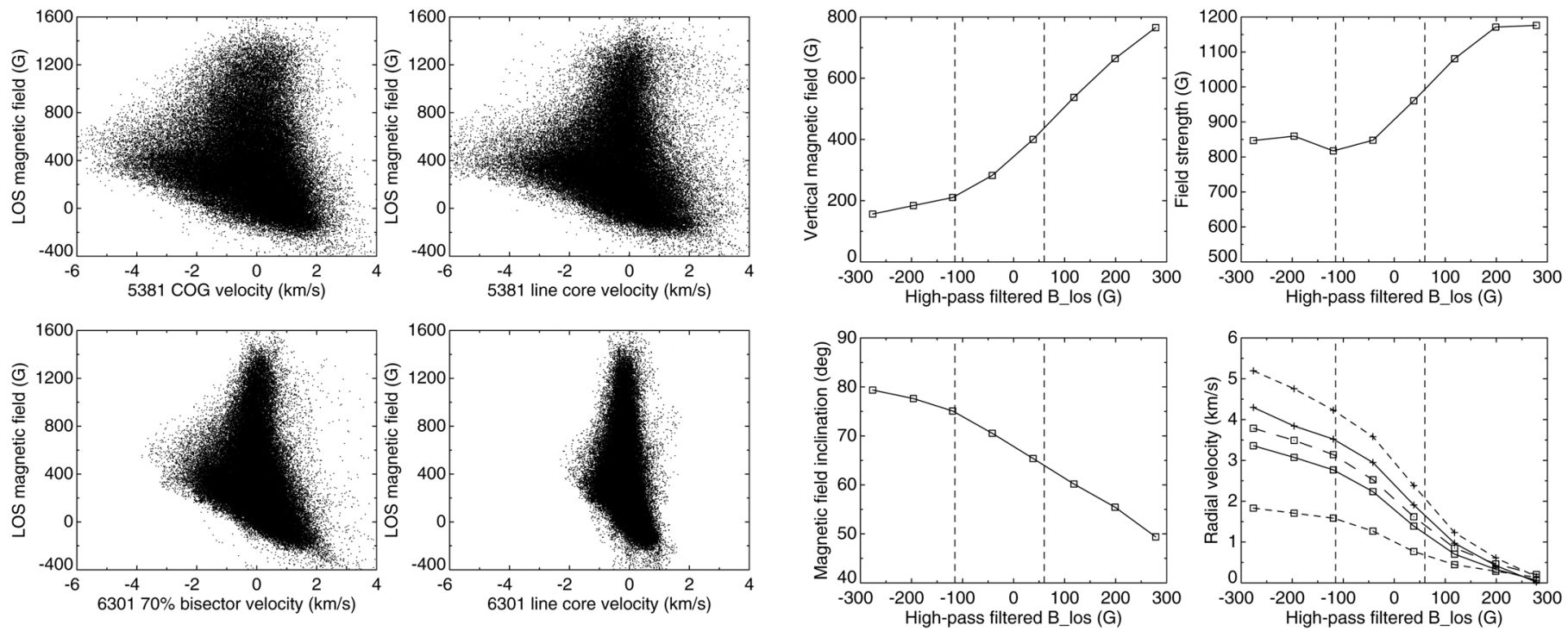

Fig. 9. Correlation between LOS velocity, measured with different methods in the 5380 and 6301 lines, and the measured LOS magnetic field. Neither of the two quantities plotted have been high-pass filtered.

flows demonstrated earlier (Scharmer et al. 2011) and here, these flow patterns would be consistent with flows in arched flux tubes, as concluded in numerous earlier interpretations of observations made at much lower spatial resolution (Solanki \& Montavon 1993; Martínez Pillet 2000; Schlichenmaier \& Collados 2002; Bellot Rubio et al. 2003, 2004; Borrero et al. 2007; Tritschler et al. 2007; Franz \& Schlichenmaier 2009) and numerical simulations (Schlichenmaier et al. 1998; Schlichenmaier 2002). As pointed out earlier (Sánchez Almeida et al. 2007; Scharmer et al. 2011) and in Sect. 2.2, such apparent large-scale penumbral flow patterns are likely to be at least partly the result of inadequate spatial resolution, giving effects similar to convective blue-shifts.

Fig. 10. Variation of "interior penumbra" (radial zones 2-4) magnetic field and radial flow speed with the strength of the high-pass filtered LOS magnetic field, obtained from azimuthal fits. The top two plots show the variation of the vertical and horizontal components of the magnetic field, the lower-left the variation in magnetic field inclination. The lower-right plot shows the (remarkable!) variation of the radial flow velocity, measured with five different methods from the 5380 and 6301 lines. Plus symbols correspond to measurements in the 5380 line, squares to the 6301 line, lines drawn full to COG measurements, short dashes to line core, and long dashes to $70 \%$ bisector (6301 line only) LOS velocities. The vertical dashed lines correspond to the upper and lower thresholds respectively, for "inter-spines" and "spines".

In Fig. 13 we show the results from azimuthal fits (Sect. 2.6) of the measured LOS velocities made at different high-passed filtered 5380 continuum intensities, separately for spines and inter-spines. The fits include pixels only from the "interior 

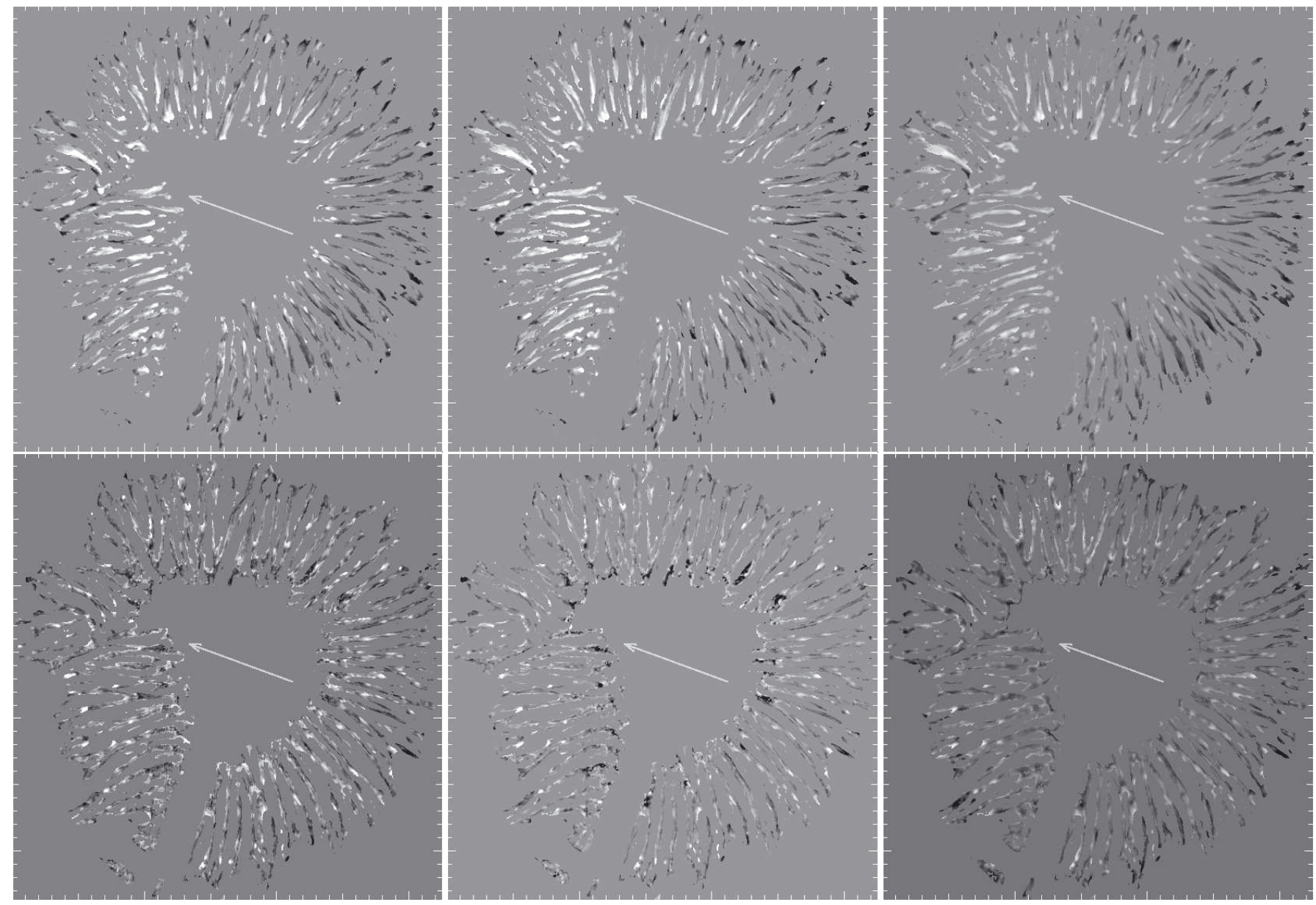

Fig. 11. Measured absolute (unfiltered) LOS velocities within the inter-spine (upper row) and spine masks (lower row) shown in Fig. 6, as obtained in the line core and with the COG method in the 5380 line and with the COG method in the 6301 line. The LOS velocities in the inter-spine mask show strong differences between the disk center and limb sides, demonstrating strong radial flows in the inter-spines, whereas no such differences are seen in the spines. Tick marks are at $1^{\prime \prime}$ intervals. The arrow points in the direction of Sun center.

penumbra", defined here as radial zones $2-4$ (see Figs. 1,2 and 5). We find strong vertical velocities that are roughly proportional to $\delta I_{\mathrm{c}}$ both in the spines and inter-spines, with the darkest structures showing downflows of up to about $1 \mathrm{~km} \mathrm{~s}^{-1}$ (except in the line core of the 6301 line). The brightest structures show upflows up to about $3 \mathrm{~km} \mathrm{~s}^{-1}$ in the 5380 line and up to $1.5 \mathrm{~km} \mathrm{~s}^{-1}$ in the 6301 line. Strong radial flows are found only in the inter-spines. The $5380 \mathrm{COG}$ measurements corroborate the earlier line core measurements made in the same line (Scharmer et al. 2011); here we detect similar clear convective signatures also in the 6301 line. The top four panels in Fig. 7 show considerable scatter around the fitted curves. This scatter shows only small variation with the azimuth angle and does not tend to disappear at $\phi=90^{\circ}$ and $\phi=270^{\circ}$, where contributions from radial flows should be absent. This clearly indicates that the scatter comes from fluctuations primarily in the vertical flows that do not correlate with intensity. As concluded already from our data, the correlation between continuum intensity and vertical velocity is somewhat weaker in the penumbra than in the quiet Sun. The same tendency is seen in simulations (Rempel 2011).

We make a few additional remarks relating to Fig. 13: first, the radial velocities obtained in the spines show a clear tendency to increase with brightness for the brightest structures. We note that Figs. 3 and 4 show several examples of bright strong flows that are located just at the boundaries of the spines. Our threshold for $\delta B_{\mathrm{LOS}}$ set to define spines is "generous" compared to that used by Scharmer et al. (2011) based on the integrated 5380 Stokes $V$ data, where the spines occupied only $13.5 \%$ of the interior penumbra. Here, we set the threshold such that spines occupy $33 \%$ of the penumbra. Increasing this threshold decreases the radial flow speed for the the brightest structures in the spines, and also reduces the strength of the radial flows at lower intensities. Second, the roughly linear relation between $\delta I_{\mathrm{c}}$ and the average vertical velocity found will not change significantly if the zero-point calibrations of LOS velocities are changed by one or two hundred $\mathrm{m} \mathrm{s}^{-1}$. This will change the value of the strongest downflows and upflows by the same amount but much larger errors would be needed to "remove" the penumbral dark downflows. Such large errors would also imply that the penumbra has an average upward velocity of well over $0.5 \mathrm{~km} \mathrm{~s}^{-1}$, in strong disagreement with earlier findings (cf., Franz 2011).

We finally note that the finding that the convective downflows are of similar strength in the 5380 and 6301 lines, while the upflows are much stronger in the 5380 line than in the 6301 line, suggests significant differences in their height variations. This is consistent with the inversions of Franz (2011), based on 6301 line data obtained with Hinode from a sunspot close to disk center. He found that a typical upflow extends only up to heights corresponding to $\log (\tau) \approx-0.5$ whereas typical downflows extend up to heights corresponding to $\log (\tau) \approx-1.2$. 
Table 1. Correlations and rms velocities.

\begin{tabular}{lccccc}
\hline \hline Quantity & $5380 \mathrm{COG}$ & 5380 core & $6301 \mathrm{COG}$ & $630170 \%$ bis. & 6301 core \\
\hline$R$ interior penumbra & -0.686 & -0.610 & -0.693 & -0.602 & -0.404 \\
$R$ quiet Sun & -0.772 & -0.796 & -0.804 & -0.734 & -0.588 \\
\hline$v_{\text {rms }}$ interior penumbra $\left(\mathrm{km} \mathrm{s}^{-1}\right)$ & 1.08 & 1.06 & 0.58 & 0.64 & 0.32 \\
$v_{\text {rms }}$ quiet Sun $\left(\mathrm{km} \mathrm{s}^{-1}\right)$ & 1.38 & 1.50 & 0.91 & 0.96 & 0.56 \\
\hline
\end{tabular}

Notes. Summary of rms velocities $\left(v_{\mathrm{rms}}\right)$ and correlation coefficients $(R)$ between high-pass spatially filtered 538 nm continuum intensities and LOS velocities $\left(v_{\text {LOS }}\right)$ measured with the 5380 and 6301 lines for quiet Sun and the interior penumbra. The different methods used for measuring the velocities are indicated above.
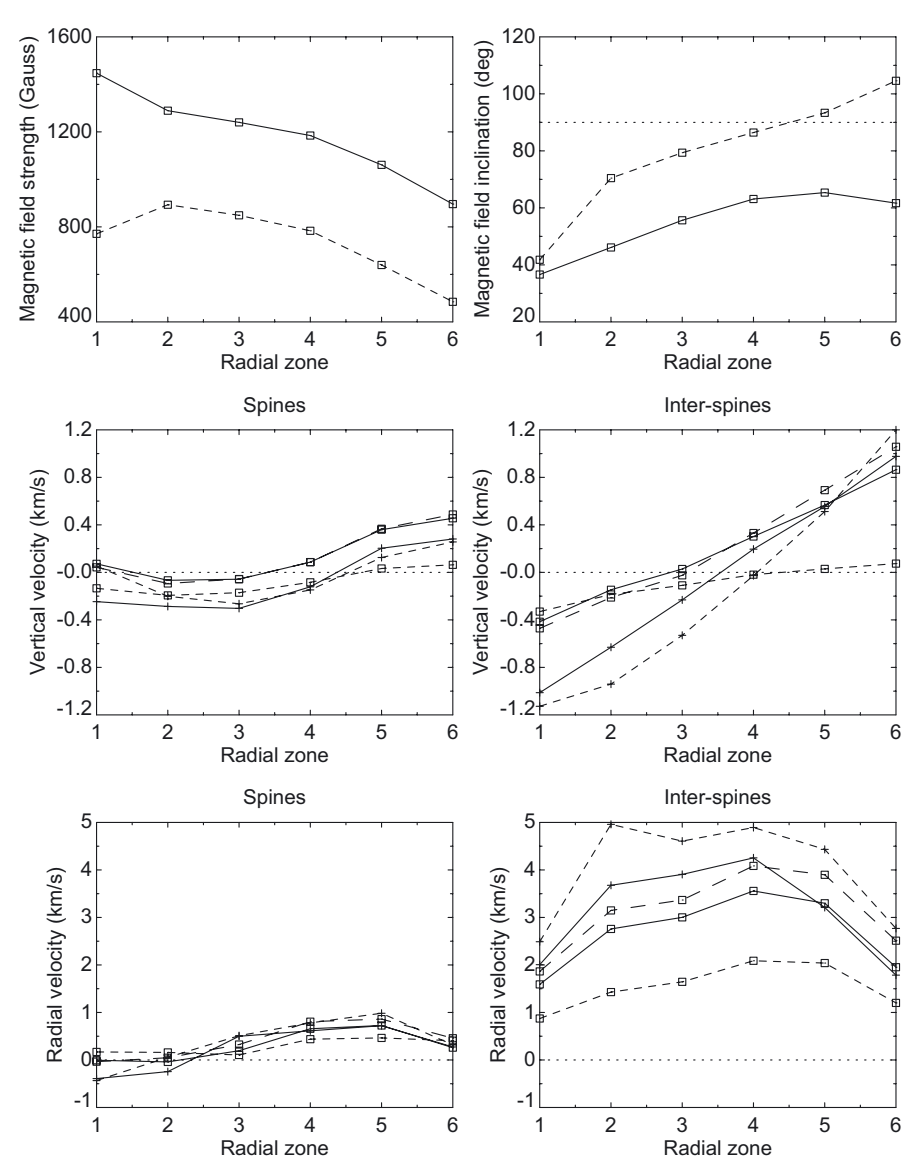

Fig. 12. Radial variation of properties of spines and inter-spines, as defined by the LOS magnetic masks, shown in Fig. 6. The plots in the top row show field strengths and inclinations for spines (full) and interspines (dashed). Symbols in the lower four plots are as in Fig. 10.

\subsection{Effects of straylight on measured vertical/radial flows}

Due to the strong weakening of the C 5380 line in dark granular and penumbral structures, straylight compensation is crucial for detecting dark convective downflows in the penumbra in this line (see SOM). The effects of straylight are less dramatic for measurements of LOS velocities in the Fe I 6301 line. Figure 14 compares the vertical and radial velocities obtained in this line with and without straylight compensation. For these fits, we included the entire interior penumbra (spines, inter-spines and the intermediate population). Except for the darkest structures, the radial velocities are similar with and without straylight compensation and (as expected) intermediate to those of spines and inter-spines, shown in Fig. 13. The strongest vertical bright upflows are reduced in strength by only a small amount
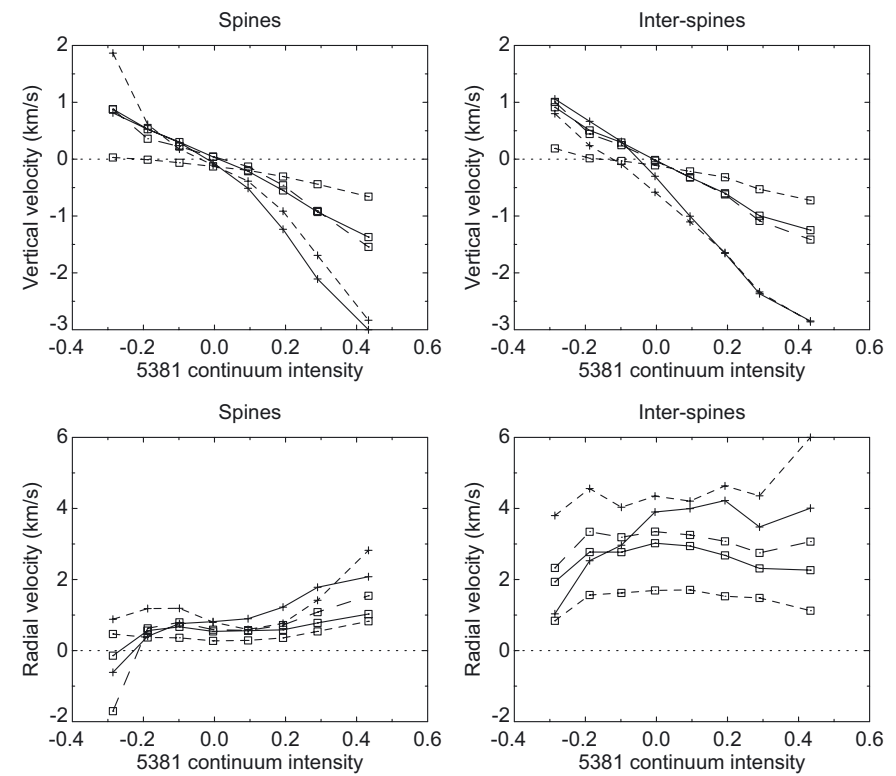

Fig. 13. Variation of vertical (top) and radial (bottom) velocities with high-pass filter $\mathrm{C}_{\mathrm{I}} 5380$ continuum intensity for spines (left) and interspines (right) in the interior penumbra (radial zones 2:4). Plus symbols correspond to measurements in the 5380 line, squares to the 6301 line, lines drawn full to COG measurements, short dashes to line core, and long dashes to $70 \%$ bisector (6301 line only) LOS velocities.

(from $1.5 \mathrm{~km} \mathrm{~s}^{-1}$ to $1.3 \mathrm{~km} \mathrm{~s}^{-1}$ ) without straylight compensation, but the darkest downflows are reduced in strength by a factor of 2 to $0.4-0.5 \mathrm{~km} \mathrm{~s}^{-1}$. Given our estimated uncertainty of $150 \mathrm{~m} \mathrm{~s}^{-1}$, we can thus establish the existence of the dark downflows from the 6301 line even without straylight compensation, but with reduced margin for error in the zero-point calibration. We conclude that both the high spatial resolution of the SST and compensation for straylight is needed for reasonably accurate estimates of the strengths of the dark convective downflows in the interior penumbra.

\section{Conclusions}

We have combined the measurements of penumbral LOS velocities in the 5380 line core reported on recently (Scharmer et al. 2011) with LOS velocity and magnetic field measurements in the 6301 line as well as COG LOS velocity measurements in the 5380 line. These observations were obtained with SST/CRISP at the highest spatial resolution $\left(0{ }^{\prime} 14\right.$ at $538 \mathrm{~nm}$ and $0{ }^{\prime} 16$ at $630 \mathrm{~nm}$ ) attainable today for spectropolarimetric data. By comparing observed quiet Sun rms continuum intensities and velocities to values established from simulations of convection, we estimate the level of straylight in our data. Constraints on the 

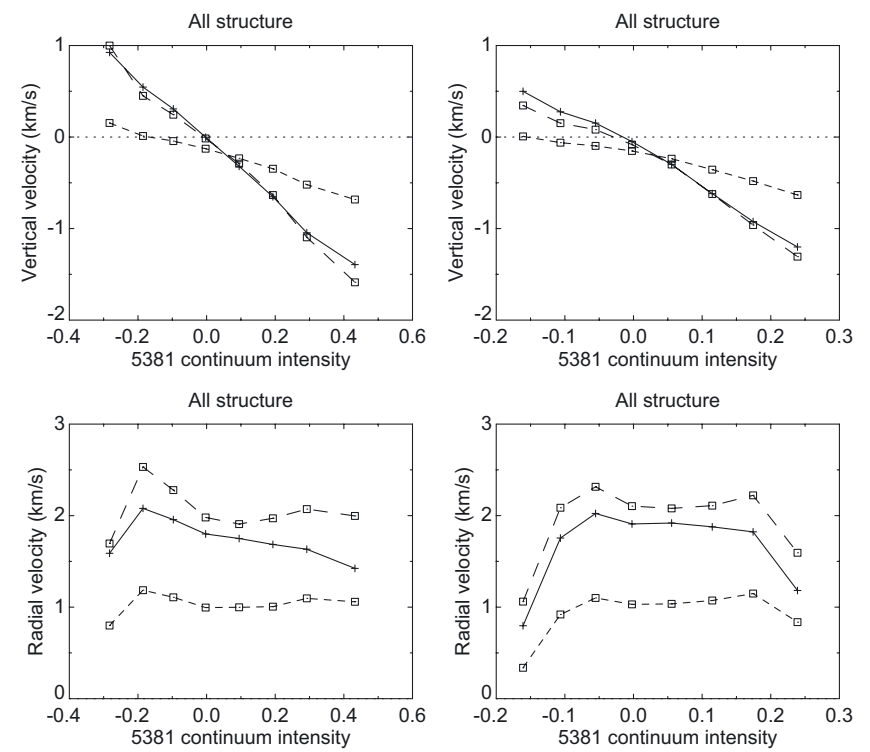

Fig. 14. Comparison of vertical (top) and radial (bottom) velocities measured in the 6301 line, with (left column) and without (right column) straylight compensation. All structure in the interior penumbra (spines, inter-spines and the intermediate population) were included in the fits. Lines drawn full correspond to COG measurements, short dashes to the line core, and long dashes to the $70 \%$ bisector LOS velocities.

FWHM of the straylight PSF are obtained from the minimum umbral intensity, which is low (15-18\%) in the observed data. By using these constraints, we compensate the data for spatial straylight (Scharmer et al. 2011).

We assume that the measured small-scale fluctuations in intensity and LOS velocity across penumbral filaments do not vary systematically with azimuth angle at constant radial distance from the inner boundary of the penumbra. Azimuthal fits of the measured LOS velocities can then be used to determine statistical properties of vertical and radial flows separately (Plaskett 1952). We have extended this widely used method (e.g., Maltby 1964; Title et al. 1993; Schlichenmaier \& Schmidt 2000; Tritschler et al. 2004; Langhans et al. 2005; Bellot Rubio et al. 2006; Sánchez Almeida et al. 2007) by making azimuthal fits separately for different (spatially filtered) continuum intensities (Scharmer et al. 2011). These fits are made for the interior penumbra (radial zones 2-4), excluding its innermost and two outermost radial zones (note that the previous analysis included radial zones 2-5, cf. SOM in Scharmer et al. 2011). We note that Sánchez Almeida et al. (2007) made azimuthal fits by assuming linear relations between local intensity fluctuations and verti$\mathrm{cal} /$ horizontal velocities. These fits demonstrated a clear correlation between intensity and the vertical velocities in the penumbra, in the sense expected for convection. However, this method cannot be used to draw conclusions about whether or not the dark component of the penumbra on the average is associated with actual downflows $\left(v_{z}>0\right)$.

Our recent analysis of line core measurements in the 5380 line demonstrated a roughly linear relation between the penumbral continuum intensity and the vertical velocity (SOM, Fig. S10). We also found that the darkest structures on the average show downflows of about $1 \mathrm{~km} \mathrm{~s}^{-1}$, whereas the brightest structures show upflows of up to $3 \mathrm{~km} \mathrm{~s}^{-1}$ in the layers corresponding to the formation height of the 5380 line (Scharmer et al. 2011). Here, we find similar downflow velocities in the dark structure but only about $1.5 \mathrm{~km} \mathrm{~s}^{-1}$ bright upflows in the wings of the 6301 line. This analysis corroborates the results obtained, and conclusions drawn from the 5380 line core measurements (Scharmer et al. 2011), but indicates that the strong bright upflows seen in the 5380 line do not extend to the heights of the 6301 line. We also find that the convective signature for vertical flows is the same irrespective of whether the magnetic field is relatively vertical and strong (in the spines) or weaker and more horizontal (in the inter-spines). However, strong radial outflows are found only in the inter-spines. This suggests the existence of either two distinct modes of (magneto-) convection simultaneously in the penumbra, where the strong Evershed flow is related to convection only in the more horizontal and weaker magnetic field structures in the inter-spines, or a more gradual change of the horizontal flow topology with magnetic field inclination.

Correlations between local fluctuations in intensity and LOS velocity in the penumbra in the sense expected for convection have been reported repeatedly in the literature since 1969 (e.g., Beckers \& Schröter 1969; Sanchez Almeida et al. 1993; Schlichenmaier \& Schmidt 1999; Schmidt \& Schlichenmaier 2000; Sánchez Almeida et al. 2007; Franz \& Schlichenmaier 2009; Franz 2011). However, the interpretation of these observations in terms of convective flows is not without ambiguity. At low spatial resolution, the large-scale structure of these flows appears to be that of an upflow of bright structure in in the inner penumbra and a downflow of dark structure in the outer parts. This flow pattern has been interpreted as support for flux tube models (Schlichenmaier \& Schmidt 1999; Schmidt \& Schlichenmaier 2000; Franz \& Schlichenmaier 2009; Franz 2011).

A major objection to the interpretation of these flows as being of convective origin is the absence of evidence of dark downflows in the interior body of the penumbra (Franz \& Schlichenmaier 2009; Franz 2011). In our analysis, we therefore exclude the outermost penumbra, where dark downflows have been reported repeatedly, from our analysis. We also exclude the innermost part of the penumbra, where bright upflows are omnipresent and have been interpreted as the footpoints of penumbral flux tubes. The discovery of dark (convective) downflows in the penumbra reported recently (Scharmer et al. 2011; Joshi et al. 2011) and the correlation between intensity and vertical flows found from the 5380 line (Scharmer et al. 2011) in the interior penumbra, is confirmed here for the 6301 line and for COG velocities in the 5380 line. The presence of intensity correlated upflows and downflows at roughly the same radial distance in the interior penumbra contradicts earlier interpretations in terms of embedded flux tubes. The present observations, combined with earlier past and recent indications from correlations between penumbral brightness and LOS velocities (e.g., Beckers \& Schröter 1969; Sánchez Almeida et al. 2007), and observed dynamics of penumbral filaments (Ichimoto et al. 2007b; Zakharov et al. 2008; Spruit et al. 2010), provides overwhelming observational evidence for interpreting the filamentary structure, dynamics, heat flux, magnetic field topology and Evershed flow of the penumbra as due to convection, rather than in terms of embedded flux tubes. This interpretation is consistent with theoretical arguments (Spruit \& Scharmer 2006; Scharmer \& Spruit 2006) as well as numerical simulations (Heinemann et al. 2007; Scharmer et al. 2008b; Rempel et al. 2009a,b; Rempel 2011).

Our observations could also be interpreted as evidence for convection rolls (Danielson 1961b), as has been suggested previously (Beckers \& Schröter 1969; Ichimoto et al. 2007b; Zakharov et al. 2008; Sánchez Almeida 2010). However, 
objections against this interpretation were raised by Scharmer (2009) on the following grounds: the convection rolls are expected to be associated with a nearly horizontal magnetic field, whereas e.g., the observations of Ichimoto et al. (2007b) refer to the inner penumbra, where the magnetic field has a strong vertical component. Also, to sustain the radiative output of a filament over its life time of on the order of an hour (Langhans et al. 2007), the convective upflows must persist to depths much larger than a few $100 \mathrm{~km}$, whereas the convective roll is a shallow phenomenon (Danielson 1961b).

The combination of high spatial resolution and straylight compensation of our data results in rms velocities for the penumbra that are on the order of $65-70 \%$ of those for the quiet Sun, such that they suffice to explain the penumbral heat flux (cf., Scharmer et al. 2011, for a more detailed discussion). This also strongly suggests that the fundamental scales at which this convection occurs is resolved in our data.

Our data also contain a wealth of information about the toplogy of the convective flows and their magnetic fields. In future work, we will investigate this in more detail. We will also compare the present data with simultaneously obtained even more highly resolved images in the $\mathrm{Ca} \mathrm{H}$ line (Henriques, in prep.). We also intend to investigate properties of penumbral dark cores (Scharmer et al. 2002), which are obvious mostly in the innermost penumbra (radial zone 1), and thus were to a large extent excluded in the present analysis. We do not exclude the possibility, however, that a small fraction of pixels within our mask defining the interior penumbra actually corresponds to dark cores, and thus are included in our azimuthal fits.

Acknowledgements. Mats Löfdahl, Dan Kiselman and Jorge Sánchez Almeida are thanked for valuable comments on the manuscript. The Swedish 1-m Solar Telescope is operated on the island of La Palma by the Institute for Solar Physics of the Royal Swedish Academy of Sciences in the Spanish Observatorio del Roque de los Muchachos of the Instituto de Astrofísica de Canarias.

\section{References}

Beckers, J. M., \& Schröter, E. H. 1969, Sol. Phys., 10, 384

Bellot Rubio, L. R., Balthasar, H., Collados, M., \& Schlichenmaier, R. 2003, A\&A, 403, L47

Bellot Rubio, L. R., Balthasar, H., \& Collados, M. 2004, A\&A, 427, 319

Bellot Rubio, L. R., Schlichenmaier, R., \& Tritschler, A. 2006, A\&A, 453, 1117

Bellot Rubio, L. R., Schlichenmaier, R., \& Langhans, K. 2010, ApJ, 725, 11

Bharti, L., Schüssler, M., \& Rempel, M. 2011, ApJ, 739, 35

Borrero, J. M., Bellot Rubio, L. R., \& Müller, D. A. N. 2007, ApJ, 666, L133

Carlsson, M., Stein, R. F., Nordlund, Å., \& Scharmer, G. B. 2004, ApJ, 610, L137

Cauzzi, G., Smaldone, L. A., Balasubramaniam, K. S., \& Keil, S. L. 1993, Sol. Phys., 146, 207

Danielson, R. E. 1961a, ApJ, 134, 275

Danielson, R. E. 1961b, ApJ, 134, 289

Danilovic, S., Gandorfer, A., Lagg, A., et al. 2008, A\&A, 484, L17

de la Cruz Rodríguez, J., Kiselman, D., \& Carlsson, M. 2011, A\&A, 528, A113

Evershed, J. 1909, MNRAS, 69, 454

Franz, M. 2011, Ph.D. Thesis [arXiv: 1107.2586]

Franz, M., \& Schlichenmaier, R. 2009, A\&A, 508, 1453

Heinemann, T., Nordlund, Å., Scharmer, G. B., \& Spruit, H. C. 2007, ApJ, 669, 1390

Ichimoto, K., Shine, R. A., Lites, B., et al. 2007a, PASJ, 59, 593

Ichimoto, K., Suematsu, Y., Tsuneta, S., et al. 2007b, Science, 318, 1597

Illing, R. M. E., Landman, D. A., \& Mickey, D. L. 1974a, A\&A, 35, 327

Illing, R. M. E., Landman, D. A., \& Mickey, D. L. 1974b, A\&A, 37, 97

Johannesson, A. 1993, A\&A, 273, 633

Joshi, J., Pietarila, A., Hirzberger, J., et al. 2011, ApJ, 734, L18

Kiselman, D. 2008, Physica Scripta Volume T, 133, 014016

Langhans, K., Scharmer, G. B., Kiselman, D., Löfdahl, M. G., \& Berger, T. E. 2005, A\&A, 436, 1087

Langhans, K., Scharmer, G. B., Kiselman, D., \& Löfdahl, M. G. 2007, A\&A, 464,763
Lites, B. W., Elmore, D. F., Seagraves, P., \& Skumanich, A. P. 1993, ApJ, 418, 928

Lites, B. W., Skumanich, A., \& Scharmer, G. B. 1990, ApJ, 355, 329

Löfdahl, M. G., \& Scharmer. 2012, A\&A, 537, A80

Maltby, P. 1964, Astrophysica Norvegica, 8, 205

Márquez, I., Sánchez Almeida, J., \& Bonet, J. A. 2006, ApJ, 638, 553

Martínez Pillet, V. 2000, A\&A, 361, 734

Mathew, S. K., Zakharov, V., \& Solanki, S. K. 2009, A\&A, 501, L19

Meyer, F., \& Schmidt, H. U. 1968, Mitteilungen der Astronomischen Gesellschaft Hamburg, 25, 194

Nordlund, A., \& Scharmer, G. B. 2010, in Magnetic Coupling between the Interior and Atmosphere of the Sun, ed. S. S. Hasan, \& R. J. Rutten, 243

Orozco Suárez, D., Bellot Rubio, L. R., Vögler, A., \& Del Toro Iniesta, J. C. 2010, A\&A, 518, A2

Plaskett, H. H. 1952, MNRAS, 112, 414

Puschmann, K. G., \& Beck, C. 2011, A\&A, 533, A21

Rees, D. E., \& Semel, M. D. 1979, A\&A, 74, 1

Rempel, M. 2011, ApJ, 729, 5

Rempel, M., Schüssler, M., Cameron, R. H., \& Knölker, M. 2009a, Science, 325, 171

Rempel, M., Schüssler, M., \& Knölker, M. 2009b, ApJ, 691, 640

Sanchez Almeida, J. 1997, ApJ, 491, 993

Sánchez Almeida, J. 2005, ApJ, 622, 1292

Sánchez Almeida, J. 2006, in ASP Conf. Ser. 358, ed. R. Casini, \& B. W. Lites, 13

Sánchez Almeida, J. 2010, in Magnetic Coupling between the Interior and Atmosphere of the Sun, ed. S. S. Hasan, \& R. J. Rutten, 210

Sanchez Almeida, J., \& Lites, B. W. 1992, ApJ, 398, 359

Sanchez Almeida, J., Martinez Pillet, V., \& Lites, J. T. B. B. W. 1993, in The Magnetic and Velocity Fields of Solar Active Regions, ed. H. Zirin, G. Ai, \& H. Wang, IAU Colloq., ASP Conf. Ser. 46, 141, 192

Sánchez Almeida, J., Márquez, I., Bonet, J. A., \& Domínguez Cerdeña, I. 2007, ApJ, 658, 1357

Scharmer, G. B. 2006, A\&A, 447, 1111

Scharmer, G. B. 2009, Space Sci. Rev., 144, 229

Scharmer, G. B., \& Spruit, H. C. 2006, A\&A, 460, 605

Scharmer, G. B., Gudiksen, B. V., Kiselman, D., Löfdahl, M. G., \& Rouppe van der Voort, L. H. M. 2002, Nature, 420, 151

Scharmer, G. B., Bjelksjö, K., Korhonen, T. K., Lindberg, B., \& Petterson, B. 2003a, in SPIE Conf. Ser., 4853, ed. S. L. Keil, \& S. V. Avakyan, 341

Scharmer, G. B., Dettori, P. M., Löfdahl, M. G., \& Shand, M. 2003b, in SPIE Conf. Ser. 4853, ed. S. L. Keil, \& S. V. Avakyan, 370

Scharmer, G. B., Narayan, G., Hillberg, T., et al. 2008a, ApJ, 689, L69

Scharmer, G. B., Nordlund, A., \& Heinemann, T. 2008b, ApJ, 677, L149

Scharmer, G. B., Löfdahl, M. G., van Werkhoven, T. I. M., \& de la Cruz Rodríguez, J. 2010, A\&A, 521, A68

Scharmer, G. B., Henriques, V. M. J., Kiselman, D., \& de la Cruz Rodríguez, J. 2011, Science, 333, 316

Schlichenmaier, R. 2002, Astron. Nachr., 323, 303

Schlichenmaier, R., \& Collados, M. 2002, A\&A, 381, 668

Schlichenmaier, R., \& Schmidt, W. 1999, A\&A, 349, L37

Schlichenmaier, R., \& Schmidt, W. 2000, A\&A, 358, 1122

Schlichenmaier, R., \& Solanki, S. K. 2003, A\&A, 411, 257

Schlichenmaier, R., Jahn, K., \& Schmidt, H. U. 1998, A\&A, 337, 897

Schmidt, W., \& Schlichenmaier, R. 2000, A\&A, 364, 829

Selbing, J. 2005, Master's Thesis, Stockholm University

Solanki, S. K., \& Montavon, C. A. P. 1993, A\&A, 275, 283

Solanki, S. K., Walther, U., \& Livingston, W. 1993, A\&A, 277, 639

Spruit, H. C., \& Scharmer, G. B. 2006, A\&A, 447, 343

Spruit, H. C., Scharmer, G. B., \& Löfdahl, M. G. 2010, A\&A, 521, A72

Stein, R. F., \& Nordlund, A. 1998, ApJ, 499, 914

Thomas, J. H., \& Montesinos, B. 1993, ApJ, 407, 398

Title, A. M., Frank, Z. A., Shine, R. A., et al. 1993, ApJ, 403, 780

Tritschler, A., Schlichenmaier, R., Bellot Rubio, L. R., et al. 2004, A\&A, 415, 717

Tritschler, A., Müller, D. A. N., Schlichenmaier, R., \& Hagenaar, H. J. 2007, ApJ, 671, L85

van Noort, M., Rouppe van der Voort, L., \& Löfdahl, M. G. 2005, Sol. Phys., 228, 191

Vargas Domínguez, S., Rouppe van der Voort, L., Bonet, J. A., et al. 2008, ApJ, 679,900

Wedemeyer-Böhm, S. 2008, A\&A, 487, 399

Wedemeyer-Böhm, S., \& Rouppe van der Voort, L. 2009, A\&A, 503, 225

Wiehr, E., \& Stellmacher, G. 1989, A\&A, 225, 528

Zakharov, V., Hirzberger, J., Riethmüller, T. L., Solanki, S. K., \& Kobel, P. 2008, A\&A, 488, L17 